\title{
STABILITY OF AN INTERCONNECTED SYSTEM OF EULER-BERNOULLI BEAM AND HEAT EQUATION WITH BOUNDARY COUPLING*
}

\author{
Jun-Min WAng ${ }^{1}$ And Miroslav Krstic ${ }^{2}$
}

\begin{abstract}
We study the stability of an interconnected system of Euler-Bernoulli beam and heat equation with boundary coupling, where the boundary temperature of the heat equation is fed as the boundary moment of the Euler-Bernoulli beam and, in turn, the boundary angular velocity of the Euler-Bernoulli beam is fed into the boundary heat flux of the heat equation. We show that the spectrum of the closed-loop system consists only of two branches: one along the real axis and the another along two parabolas symmetric to the real axis and open to the imaginary axis. The asymptotic expressions of both eigenvalues and eigenfunctions are obtained. With a careful estimate for the resolvent operator, the completeness of the root subspaces of the system is verified. The Riesz basis property and exponential stability of the system are then proved. Finally we show that the semigroup, generated by the system operator, is of Gevrey class $\delta>2$.
\end{abstract}

Mathematics Subject Classification. 93D15, 93C20, 35P20.

Received January 23, 2013. Revised January 4, 2014.

Published online June 19, 2015.

\section{INTRODUCTION}

Engineering applications give rise to fluid-structure interactions, composite laminates in smart materials and structures, structural-acoustic systems, and other interactive physical process, which are modeled by partial differential equation (PDE) cascades or interconnected PDEs. Control design and stability analysis for such systems have become active over the past decades, see $[5,6,8,19,20,23,24]$ and the references therein.

The stability and controllability analysis for a heat-wave system, arising from the fluid-structure interaction, were treated in [23,24]. Feedback controllers for several classes of coupled PDEs and structural-acoustic models were introduced in [8]. The stability and Riesz basis property of the composite laminates and the sandwich beam with boundary controls were analyzed in $[19,20]$.

\footnotetext{
Keywords and phrases. Euler-Bernoulli beam, heat equation, boundary control, stability, spectrum, Gevrey regularity.

* The research was supported by the National Natural Science Foundation of China.

1 School of Mathematics and Statistics, Beijing Institute of Technology, Beijing 100081, P.R. China. jmwang@bit.edu.cn

2 Department of Mechanical and Aerospace Engineering, University of California at San Diego, La Jolla, CA 92093-0411, USA. krstic@ucsd.edu
} 


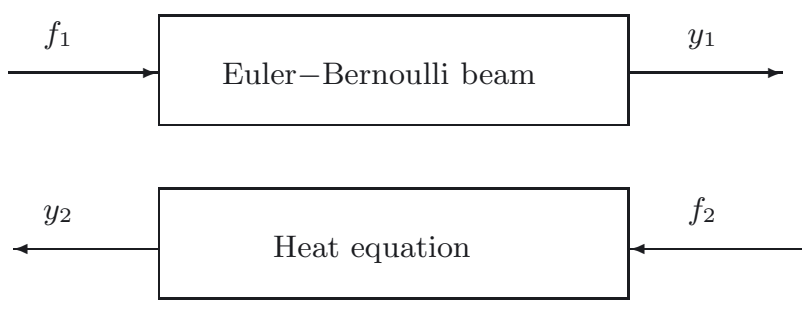

Figure 1. Euler-Bernoulli beam (1.1) and heat equation (1.2).

We consider Euler-Bernoulli beam and heat equation (see Fig. 1) governed by the equations:

$$
\text { Euler-Bernoulli beam : } \begin{cases}w_{t t}(x, t)+w_{x x x x}(x, t)=0, & 0<x<1, t>0, \\ w(0, t)=w(1, t)=w_{x x}(1, t)=0, & t \geq 0, \\ w_{x x}(0, t)=f_{1}(t), & t \geq 0, \\ y_{1}(t)=-w_{x t}(0, t), & t \geq 0, \\ w(x, 0)=w_{0}(x), w_{t}(x, 0)=w_{1}(x), 0 \leq x \leq 1,\end{cases}
$$

and

$$
\text { Heat equation : } \begin{cases}u_{t}(x, t)-u_{x x}(x, t)=0,0<x<1, t>0, \\ u(1, t)=0, & t \geq 0, \\ u_{x}(0, t)=f_{2}(t), & t \geq 0, \\ y_{2}(t)=-u(0, t), & t \geq 0, \\ u(x, 0)=u_{0}(x), & 0 \leq x \leq 1,\end{cases}
$$

where the Euler-Bernoulli beam is hinged at the right hand, the right side of the heat equation is kept at zero temperature, $f_{1}(t)$ and $f_{2}(t)$ are the boundary controls applied at the left ends of the beam and the heat respectively, $y_{1}(t)$ and $y_{2}(t)$ are the observations, and $\left(w_{0}(x), w_{1}(x)\right)$ and $u_{0}(x)$ are the initial conditions. We denote the two dynamic systems with the mappings

$$
\mathfrak{E}: f_{1} \mapsto y_{1}
$$

and

$$
\mathfrak{H}: f_{2} \mapsto y_{2}
$$

It is well-known that the feedback law

$$
f_{1}(t)=-y_{1}(t)
$$

achieves exponential stability of the Euler-Bernoulli beam system, as well as that the feedback law

$$
f_{2}(t)=-y_{2}(t)
$$

guarantees exponential stability of the heat equation. In this paper we study the case where the two subsystems are interconnected via the feedback laws (see Fig. 2)

$$
f_{1}(t)=-y_{2}(t)
$$




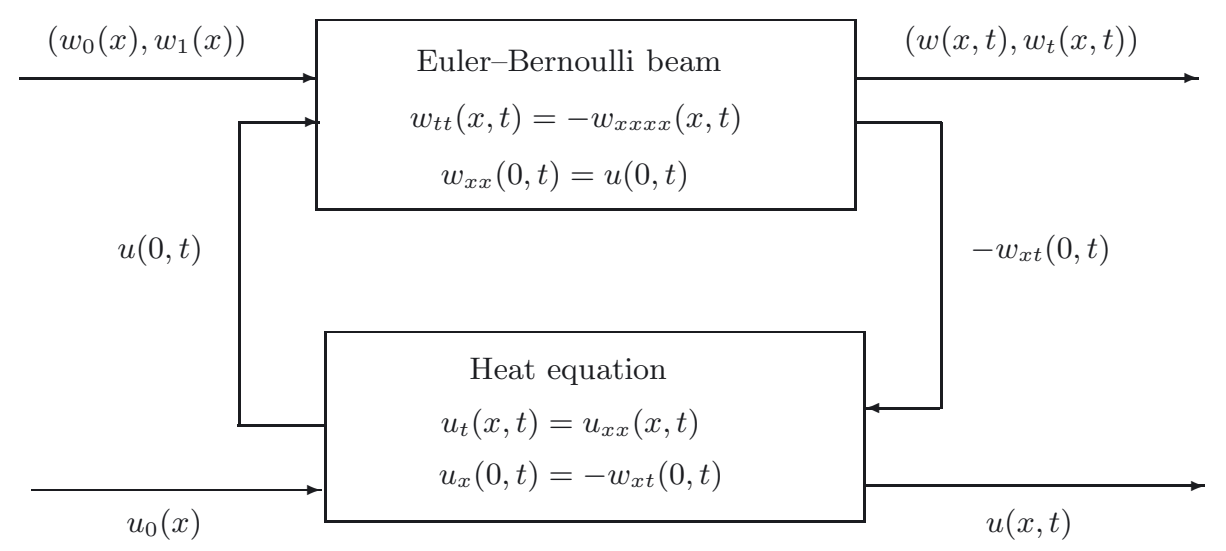

FiguRE 2. Block diagram for the closed-loop system (1.7).

and

$$
f_{2}(t)=y_{1}(t)
$$

The interconnection (1.5) and (1.6) can be interpreted in three ways. The first interpretation of (1.5) and (1.6) is as

$$
f_{1}(t)=\left(-\mathfrak{H} y_{1}\right)(t)
$$

namely, as replacing the unit-gain static feedback (1.3) of the Euler-Bernoulli beam by a dynamic feedback law governed by the heat equation. The second interpretation of (1.5) and (1.6) is as

$$
f_{2}(t)=\left(\mathfrak{E}\left(-y_{2}\right)\right)(t),
$$

namely, as replacing the unity-gain static feedback (1.4) of the heat equation by a dynamic feedback law governed by the Euler-Bernoulli beam. The third interpretation of (1.5), (1.6) is simply as a coupled PDE system given in Figure 2.

Under the feedback laws (1.5), (1.6), the interconnected system of Euler-Bernoulli beam and heat equation is:

$$
\begin{cases}w_{t t}(x, t)+w_{x x x x}(x, t)=0, & 0<x<1, t>0, \\ u_{t}(x, t)-u_{x x}(x, t)=0, & 0<x<1, t>0, \\ w(1, t)=w_{x x}(1, t)=0, & t \geq 0, \\ u(1, t)=0, & t \geq 0, \\ w(0, t)=0, & t \geq 0, \\ w_{x x}(0, t)=u(0, t), & t \geq 0, \\ u_{x}(0, t)=-w_{x t}(0, t), & t \geq 0, \\ w(x, 0)=w_{0}(x), w_{t}(x, 0)=w_{1}(x), & 0 \leq x \leq 1, \\ u(x, 0)=u_{0}(x), & 0 \leq x \leq 1 .\end{cases}
$$

The energy function for (1.7) is given by

$$
E(t)=\frac{1}{2} \int_{0}^{1}\left[w_{t}^{2}(x, t)+w_{x x}^{2}(x, t)+u^{2}(x, t)\right] \mathrm{d} x .
$$


Then we have

$$
\begin{aligned}
\frac{\mathrm{d}}{\mathrm{d} t} E(t) & =\int_{0}^{1}\left[w_{t}(x, t) w_{t t}(x, t)+w_{x x}(x, t) w_{x x t}(x, t)+u(x, t) u_{t}(x, t)\right] \mathrm{d} x \\
& =\int_{0}^{1}\left[-w_{t}(x, t) w_{x x x x}(x, t)+w_{x x}(x, t) w_{x x t}(x, t)+u(x, t) u_{x x}(x, t)\right] \mathrm{d} x \\
& =-\left.w_{t} w_{x x x}\right|_{0} ^{1}+\left.w_{x t} w_{x x}\right|_{0} ^{1}+\left.u u_{x}\right|_{0} ^{1}-\int_{0}^{1} u_{x}^{2}(x, t) \mathrm{d} x=-\int_{0}^{1} u_{x}^{2}(x, t) \mathrm{d} x \leq 0
\end{aligned}
$$

and $E(t)$ is non-increasing.

We provide a detailed spectral analysis for the system (1.7). We show that there are two branches of eigenvalues of (1.7): one is along the real axis, and another is along the two parabolas symmetric to the real axis and open to the imaginary axis. The latter branch of eigenvalues generated by the beam is very similar to the case studied in [4], where the well-posedness and exponential stability of an Euler-Bernoulli beam with nonmonotone boundary feedback $w_{x x x}(0, t)=-k w_{x t}(0, t)$, proposed early in [11], were considered for the feedback gain $k>0$ with $k \neq 1$. Later on, its Gevrey regularity was treated in $[1,14,17]$.

In this paper, the asymptotic expressions of the eigenvalues and eigenfunctions, the Riesz basis property and exponential stability of (1.7) are studied. Moreover, we show that the $C_{0}$-semigroup, generated by the system operator, is of Gevrey class $\delta>2$ (Gevrey regularity is described in terms of the bounds on all derivatives of the semigroups. The differentiability of the Gevrey semigroup is slightly weaker than that of an analytic semigroup $[1,14,17,18])$. The Gevrey regularity for a Schrödinger equation in boundary feedback with a heat equation is obtained in [21].

We proceed as follows. In Section 2 we formulate the problem as an evolution equation in Hilbert energy space. The $C_{0}$-semigroup approach is used to prove the well-posedness of the system. Section 3 is devoted to the spectral analysis and the asymptotic expressions of eigenvalues and eigenfunctions are presented. By estimating the resolvent operator of the system, the completeness of the root subspace of the system is proved in Section 4 . In Section 5, the Riesz basis property and exponential stability are established. Finally, Gevrey regularity of the semigroup is obtained in Section 6 .

\section{WeLL-POSEDNESS OF THE SYSTEM (1.7)}

We consider the system (1.7) in the energy space

$$
\mathcal{H}=H_{L}^{2}(0,1) \times L^{2}(0,1) \times L^{2}(0,1)
$$

where $H_{L}^{2}(0,1)=\left\{f \mid f \in H^{2}(0,1), f(0)=f(1)=0\right\}$ and the norm in $\mathcal{H}$ is induced by the following inner product

$$
\left\langle X_{1}, X_{2}\right\rangle=\int_{0}^{1}\left[f_{1}^{\prime \prime}(x) \overline{f_{2}^{\prime \prime}(x)}+g_{1}(x) \overline{g_{2}(x)}+h_{1}(x) \overline{h_{2}(x)}\right] \mathrm{d} x,
$$

where $X_{i}=\left(f_{i}, g_{i}, h_{i}\right) \in \mathcal{H}, i=1,2$. Define the system operator by

$$
\left\{\begin{array}{l}
\mathcal{A}(f, g, h)=\left(g,-f^{(4)}, h^{\prime \prime}\right), \forall(f, g, h) \in D(\mathcal{A}), \\
D(\mathcal{A})=\left\{(f, g, h) \in\left(H^{4} \times H_{L}^{2} \times H^{2}\right) \cap \mathcal{H} \mid \begin{array}{l}
h(1)=f^{\prime \prime}(1)=0, \\
g^{\prime}(0)=-h^{\prime}(0), f^{\prime \prime}(0)=h(0)
\end{array}\right\} .
\end{array}\right.
$$

Then (1.7) can be written as an evolution equation in $\mathcal{H}$ :

$$
\left\{\begin{array}{l}
\frac{\mathrm{d} X(t)}{\mathrm{d} t}=\mathcal{A} X(t), t>0, \\
X(0)=X_{0},
\end{array}\right.
$$

where $X(t)=\left(w(\cdot, t), w_{t}(\cdot, t), u(\cdot, t)\right)$ and $X_{0}=\left(w_{0}, w_{1}, u_{0}\right)$. 
Theorem 2.1. Let $\mathcal{A}$ be given by (2.1). Then $\mathcal{A}^{-1}$ exists and is compact. Moreover $\mathcal{A}$ is dissipative in $\mathcal{H}$ and $\mathcal{A}$ generates a $C_{0}$-semigroup $\mathrm{e}^{\mathcal{A} t}$ of contractions in $\mathcal{H}$.

Proof. For any given $\left(f_{1}, g_{1}, h_{1}\right) \in \mathcal{H}$, solve

$$
\mathcal{A}(f, g, h)=\left(g,-f^{(4)}, h^{\prime \prime}\right)=\left(f_{1}, g_{1}, h_{1}\right) .
$$

We get $g(x)=f_{1}(x)$ directly. To get $h$ we solve

$$
\left\{\begin{array}{l}
h^{\prime \prime}(x)=h_{1}(x) \\
h(1)=0, h^{\prime}(0)=-g^{\prime}(0)=-f_{1}^{\prime}(0)
\end{array}\right.
$$

obtaining

$$
h(x)=f_{1}^{\prime}(0)(1-x)-\left[\int_{0}^{x}(1-x) h_{1}(\xi) \mathrm{d} \xi+\int_{x}^{1}(1-\xi) h_{1}(\xi) \mathrm{d} \xi\right] .
$$

To get $f$ we solve

$$
\left\{\begin{array}{l}
f^{(4)}(x)=-g_{1}(x) \\
f(0)=f(1)=f^{\prime \prime}(1)=0, f^{\prime \prime}(0)=h(0)
\end{array}\right.
$$

obtaining

$$
\left\{\begin{array}{l}
f(x)=\int_{0}^{x} \varphi(s)(x-s) \mathrm{d} s-x \int_{0}^{1} \varphi(s)(1-s) \mathrm{d} s \\
\varphi(x)=\int_{0}^{x} g_{1}(s)(s-x) \mathrm{d} s+x \int_{0}^{1} g_{1}(s)(1-s) \mathrm{d} s+h(0)(1-x) \\
h(0)=f_{1}^{\prime}(0)-\int_{0}^{1}(1-\xi) h_{1}(\xi) \mathrm{d} \xi
\end{array}\right.
$$

By $(2.3),(2.4)$ and $g(x)=f_{1}(x)$, we get the unique $(f, g, h) \in D(\mathcal{A})$. Hence, $\mathcal{A}^{-1}$ exists and is compact on $\mathcal{H}$ by the Sobolev embedding theorem. Now we show that $\mathcal{A}$ is dissipative in $\mathcal{H}$. Let $X=(f, g, h) \in D(\mathcal{A})$. Then we have

$$
\begin{aligned}
\langle\mathcal{A} X, X\rangle & =\left\langle\left(g,-f^{(4)}, h^{\prime \prime}\right),(f, g, h)\right\rangle=\int_{0}^{1} g^{\prime \prime} \overline{f^{\prime \prime}} \mathrm{d} x-\int_{0}^{1} f^{(4)} \bar{g} \mathrm{~d} x+\int_{0}^{1} h^{\prime \prime} \bar{h} \mathrm{~d} x \\
& =-\left.f^{\prime \prime \prime} \bar{g}\right|_{0} ^{1}+\left.f^{\prime \prime} \overline{g^{\prime}}\right|_{0} ^{1}+\left.h^{\prime} \bar{h}\right|_{0} ^{1}+\int_{0}^{1} g^{\prime \prime} \overline{f^{\prime \prime}} \mathrm{d} x-\int_{0}^{1} f^{\prime \prime} \overline{g^{\prime \prime}} \mathrm{d} x-\int_{0}^{1}\left|h^{\prime}\right|^{2} \mathrm{~d} x \\
& =-f^{\prime \prime}(0) \overline{g^{\prime}(0)}-h^{\prime}(0) \overline{h(0)}+\int_{0}^{1} g^{\prime \prime} \overline{f^{\prime \prime}} \mathrm{d} x-\int_{0}^{1} f^{\prime \prime} \overline{g^{\prime \prime}} \mathrm{d} x-\int_{0}^{1}\left|h^{\prime}\right|^{2} \mathrm{~d} x \\
& =\int_{0}^{1} g^{\prime \prime} \overline{f^{\prime \prime}} \mathrm{d} x-\int_{0}^{1} f^{\prime \prime} \overline{g^{\prime \prime}} \mathrm{d} x-\int_{0}^{1}\left|h^{\prime}\right|^{2} \mathrm{~d} x
\end{aligned}
$$

and

$$
\operatorname{Re}\langle\mathcal{A} X, X\rangle=-\int_{0}^{1}\left|h^{\prime}\right|^{2} \mathrm{~d} x \leq 0
$$

Hence $\mathcal{A}$ is dissipative and $\mathcal{A}$ generates a $C_{0}$-semigroup $\mathrm{e}^{\mathcal{A} t}$ of contractions in $\mathcal{H}$ by the Lumer-Philips Theorem [15].

Remark 2.2. As an elementary consequence of the compactness of $\mathcal{A}^{-1}, \sigma(\mathcal{A})$, the spectrum of $\mathcal{A}$, consists of isolated eigenvalues of finite algebraic multiplicity only. 


\section{Spectral ANALYSis}

Let us now consider the eigenvalue problem of $\mathcal{A}$. $\mathcal{A} X=\lambda X$, where $X=(f, g, h) \in D(\mathcal{A})$, if and only if $g(x)=\lambda f(x)$, and $f, h$ satisfy the following eigenvalue problem:

$$
\left\{\begin{array}{l}
f^{(4)}(x)+\lambda^{2} f(x)=0, \\
h^{\prime \prime}(x)-\lambda h(x)=0, \\
f(0)=f(1)=f^{\prime \prime}(1)=h(1)=0, \\
f^{\prime \prime}(0)=h(0), \\
\lambda f^{\prime}(0)=-h^{\prime}(0) .
\end{array}\right.
$$

Lemma 3.1. Let $\mathcal{A}$ be defined by (2.1). Then for each $\lambda \in \sigma(\mathcal{A})$, we have $\operatorname{Re} \lambda<0$.

Proof. By Theorem 2.1, since $\mathcal{A}$ is dissipative, we have for each $\lambda \in \sigma(\mathcal{A}), \operatorname{Re} \lambda \leq 0$. So we only need to show there are no eigenvalues on the imaginary axis. Let $\lambda=i \mu^{2} \in \sigma(\mathcal{A})$ with $\mu \in \mathbb{R}^{+}$and $X=(f, g, h) \in D(\mathcal{A})$ be its associated eigenfunction of $\mathcal{A}$. Then by (2.5), we have

$$
\operatorname{Re}\langle\mathcal{A} X, X\rangle=\operatorname{Re}\left(i \mu^{2}\langle X, X\rangle\right)=-\int_{0}^{1}\left|h^{\prime}\right|^{2} \mathrm{~d} x=0
$$

and hence $h^{\prime}(x)=0$. By $h(1)=0$, we have $h=0$. Moreover $\mathcal{A} X=i \mu^{2} X$ further gives that $g=i \mu^{2} f$ and $f$ satisfies the following

$$
\left\{\begin{array}{l}
f^{(4)}(x)-\mu^{4} f(x)=0, \\
f(0)=f(1)=f^{\prime \prime}(0)=f^{\prime \prime}(1)=f^{\prime}(0)=0 .
\end{array}\right.
$$

A direct computation yields that the above equation only has the trivial solution. Hence $f=g=h=0$ and $X=0$. Therefore, there are no eigenvalues on the imaginary axis.

Due to Lemma 3.1 and the fact that the eigenvalues are symmetric about the real axis, we consider only those $\lambda$ which are located in the second quadrant of the complex plane:

$$
\lambda:=i \rho^{2}, \rho \in \mathcal{S}:=\left\{\rho \in \mathbb{C} \mid 0 \leq \arg \rho \leq \frac{\pi}{4}\right\} .
$$

Note that for any $\rho \in \mathcal{S}$, we have

$$
\operatorname{Re}(-\rho) \leq \operatorname{Re}(i \rho) \leq \operatorname{Re}(-i \rho) \leq \operatorname{Re}(\rho)
$$

and

$$
\left\{\begin{array}{l}
\operatorname{Re}(-\rho)=-|\rho| \cos (\arg \rho) \leq-\frac{\sqrt{2}}{2}|\rho|<0, \\
\operatorname{Re}(i \rho)=-|\rho| \sin (\arg \rho) \leq 0 .
\end{array}\right.
$$

Moreover, if we denote $\mathcal{S}=\mathcal{S}_{1} \cup \mathcal{S}_{2}$ with

$$
\left\{\begin{array}{l}
\mathcal{S}_{1}:=\left\{\rho \in \mathbb{C} \mid \frac{\pi}{8}<\arg \rho \leq \frac{\pi}{4}\right\}, \\
\mathcal{S}_{2}:=\left\{\rho \in \mathbb{C} \mid 0 \leq \arg \rho \leq \frac{\pi}{8}\right\},
\end{array}\right.
$$

then we have

$$
\left\{\begin{array}{l}
\operatorname{Re}(i \rho)=-|\rho| \sin (\arg \rho) \leq-|\rho| \sin \left(\frac{1}{8} \pi\right)<0, \\
\operatorname{Re}(-\sqrt{\mathrm{i}} \rho)=-|\rho| \cos \left(\frac{\pi}{4}+\arg \rho\right) \leq-|\rho| \cos \left(\frac{3}{8} \pi\right)<0, \forall \rho \in \mathcal{S}_{1},
\end{array}\right.
$$


Now substituting $\lambda=i \rho^{2}$ into (3.1), we have the eigenvalue system of (1.7) in $\rho$ :

$$
\left\{\begin{array}{l}
f^{(4)}(x)-\rho^{4} f(x)=0 \\
h^{\prime \prime}(x)-i \rho^{2} h(x)=0 \\
f(0)=f(1)=f^{\prime \prime}(1)=h(1)=0 \\
f^{\prime \prime}(0)=h(0) \\
i \rho^{2} f^{\prime}(0)=-h^{\prime}(0)
\end{array}\right.
$$

Let

$$
f(x)=c_{1} \mathrm{e}^{\rho x}+c_{2} \mathrm{e}^{-\rho x}+c_{3} \mathrm{e}^{\mathrm{i} \rho x}+c_{4} \mathrm{e}^{-\mathrm{i} \rho x}, \quad h(x)=d_{1} \mathrm{e}^{\sqrt{\mathrm{i}} \rho x}+d_{2} \mathrm{e}^{-\sqrt{\mathrm{i}} \rho x},
$$

where $c_{s}, s=1,2,3,4, d_{1}, d_{2}$ are constants, and $\sqrt{i}=\mathrm{e}^{\mathrm{i} \frac{\pi}{4}}=\frac{\sqrt{2}}{2}(1+i)$. Substituting these into the boundary conditions of (3.7), we have

$$
\left\{\begin{array}{l}
c_{1}+c_{2}+c_{3}+c_{4}=0, \\
c_{1} \mathrm{e}^{\rho}+c_{2} \mathrm{e}^{-\rho}+c_{3} \mathrm{e}^{\mathrm{i} \rho}+c_{4} \mathrm{e}^{-\mathrm{i} \rho}=0, \\
c_{1} \rho^{2} \mathrm{e}^{\rho}+c_{2} \rho^{2} \mathrm{e}^{-\rho}-c_{3} \rho^{2} \mathrm{e}^{\mathrm{i} \rho}-c_{4} \rho^{2} \mathrm{e}^{-\mathrm{i} \rho}=0, \\
d_{1} \mathrm{e}^{\sqrt{\mathrm{i}} \rho}+d_{2} \mathrm{e}^{-\sqrt{\mathrm{i}} \rho}=0, \\
c_{1} \rho^{2}+c_{2} \rho^{2}-c_{3} \rho^{2}-c_{4} \rho^{2}-d_{1}-d_{2}=0, \\
c_{1} i \rho^{3}-c_{2} i \rho^{3}-c_{3} \rho^{3}+c_{4} \rho^{3}+d_{1} \sqrt{i} \rho-d_{2} \sqrt{i} \rho=0 .
\end{array}\right.
$$

Then (3.7) has the nontrivial solution if and only if the characteristic determinant $\operatorname{det} \Delta(\rho)=0$, where

$$
\Delta(\rho)=\left[\begin{array}{cccccc}
1 & 1 & 1 & 1 & 0 & 0 \\
\mathrm{e}^{\rho} & \mathrm{e}^{-\rho} & \mathrm{e}^{\mathrm{i} \rho} & \mathrm{e}^{-\mathrm{i} \rho} & 0 & 0 \\
\rho^{2} \mathrm{e}^{\rho} & \rho^{2} \mathrm{e}^{-\rho} & -\rho^{2} \mathrm{e}^{\mathrm{i} \rho} & -\rho^{2} \mathrm{e}^{-\mathrm{i} \rho} & 0 & 0 \\
0 & 0 & 0 & 0 & \mathrm{e}^{\sqrt{\mathrm{i}} \rho} & \mathrm{e}^{-\sqrt{\mathrm{i}} \rho} \\
\rho^{2} & \rho^{2} & -\rho^{2} & -\rho^{2} & -1 & -1 \\
i \rho^{3} & -i \rho^{3} & -\rho^{3} & \rho^{3} & \sqrt{i} \rho & -\sqrt{\mathrm{i} \rho}
\end{array}\right] .
$$

Lemma 3.2. Let $\lambda=i \rho^{2}$ with $\rho \in \mathcal{S}$ and let $\Delta(\rho)$ be given by (3.9). Then the following asymptotic expansion holds:

$$
\operatorname{det} \Delta(\rho)=-2 \rho^{5} \mathrm{e}^{\rho}\left\{a_{1} \mathrm{e}^{\mathrm{i} \rho} \mathrm{e}^{\sqrt{\mathrm{i} \rho}}+a_{2} \mathrm{e}^{\mathrm{i} \rho} \mathrm{e}^{-\sqrt{\mathrm{i} \rho}}+a_{3} \mathrm{e}^{-\mathrm{i} \rho} \mathrm{e}^{\sqrt{\mathrm{i}} \rho}+a_{4} \mathrm{e}^{-\mathrm{i} \rho} \mathrm{e}^{-\sqrt{\mathrm{i} \rho}}+\mathcal{O}\left(\mathrm{e}^{-|\rho|}\right)\right\},
$$

where

$$
\left\{\begin{array}{l}
a_{1}=1+\sqrt{2}+i(1+\sqrt{2}), a_{2}=\sqrt{2}-1+i(\sqrt{2}-1), \\
a_{3}=1-\sqrt{2}-i(1+\sqrt{2}), a_{4}=-1-\sqrt{2}-i(\sqrt{2}-1) .
\end{array}\right.
$$

Moreover, we have a more accurate asymptotic expansion, that is, when $\rho \in \mathcal{S}_{1}$ and $\rho \in \mathcal{S}_{2}$, $\operatorname{det} \Delta(\rho)$ has more accurate asymptotic expansions respectively,

$$
\operatorname{det} \Delta(\rho)=-2 \rho^{5} \mathrm{e}^{\rho} \mathrm{e}^{-\mathrm{i} \rho}\left\{a_{3} \mathrm{e}^{\sqrt{\mathrm{i}} \rho}+a_{4} \mathrm{e}^{-\sqrt{\mathrm{i}} \rho}+\mathcal{O}\left(\mathrm{e}^{-k_{1}|\rho|}\right)\right\}, \quad \rho \in \mathcal{S}_{1},
$$

and

$$
\operatorname{det} \Delta(\rho)=-2 \rho^{5} \mathrm{e}^{\rho} \mathrm{e}^{\sqrt{\mathrm{i}} \rho}\left\{a_{1} \mathrm{e}^{\mathrm{i} \rho}+a_{3} \mathrm{e}^{-\mathrm{i} \rho}+\mathcal{O}\left(\mathrm{e}^{-k_{2}|\rho|}\right)\right\}, \quad \rho \in \mathcal{S}_{2}
$$

where $k_{1}$ and $k_{2}$ are positive constants. 
Proof. From (3.9), a direct computation gives

$$
\begin{aligned}
& \operatorname{det} \Delta(\rho)=-\left|\begin{array}{cc}
\mathrm{e}^{\sqrt{\mathrm{i}} \rho} & \mathrm{e}^{-\sqrt{\mathrm{i}} \rho} \\
1 & 1
\end{array}\right|\left|\begin{array}{cccc}
1 & 1 & 1 & 1 \\
\mathrm{e}^{\rho} & \mathrm{e}^{-\rho} & \mathrm{e}^{\mathrm{i} \rho} & \mathrm{e}^{-\mathrm{i} \rho} \\
\rho^{2} \mathrm{e}^{\rho} & \rho^{2} \mathrm{e}^{-\rho} & -\rho^{2} \mathrm{e}^{\mathrm{i} \rho} & -\rho^{2} \mathrm{e}^{-\mathrm{i} \rho} \\
i \rho^{3} & -i \rho^{3} & -\rho^{3} & \rho^{3}
\end{array}\right| \\
& -\left|\begin{array}{cccc}
\mathrm{e}^{\sqrt{\mathrm{i}} \rho} & \mathrm{e}^{-\sqrt{\mathrm{i}} \rho} \\
\sqrt{i} \rho-\sqrt{\mathrm{i}} \rho
\end{array}\right|\left|\begin{array}{cccc}
1 & 1 & 1 & 1 \\
\mathrm{e}^{\rho} & \mathrm{e}^{-\rho} & \mathrm{e}^{\mathrm{i} \rho} & \mathrm{e}^{-\mathrm{i} \rho} \\
\rho^{2} \mathrm{e}^{\rho} & \rho^{2} \mathrm{e}^{-\rho} & -\rho^{2} \mathrm{e}^{\mathrm{i} \rho} & -\rho^{2} \mathrm{e}^{-\mathrm{i} \rho} \\
\rho^{2} & \rho^{2} & -\rho^{2} & -\rho^{2}
\end{array}\right| \\
& =-\rho^{5}\left[\mathrm{e}^{\sqrt{\mathrm{i}} \rho}-\mathrm{e}^{-\sqrt{\mathrm{i}} \rho}\right] G_{1}(\rho)+\sqrt{i} \rho^{5}\left[\mathrm{e}^{\sqrt{\mathrm{i}} \rho}+\mathrm{e}^{-\sqrt{\mathrm{i}} \rho}\right] G_{2}(\rho),
\end{aligned}
$$

where

$$
G_{1}(\rho)=\left|\begin{array}{cccc}
1 & 1 & 1 & 1 \\
\mathrm{e}^{\rho} \mathrm{e}^{-\rho} & \mathrm{e}^{\mathrm{i} \rho} & \mathrm{e}^{-\mathrm{i} \rho} \\
\mathrm{e}^{\rho} \mathrm{e}^{-\rho} & -\mathrm{e}^{\mathrm{i} \rho} & -\mathrm{e}^{-\mathrm{i} \rho} \\
i & -i & -1 & 1
\end{array}\right|=2 \mathrm{e}^{\rho}\left[(1+i) \mathrm{e}^{\mathrm{i} \rho}+(1-i) \mathrm{e}^{-\mathrm{i} \rho}+\mathcal{O}\left(\mathrm{e}^{-\rho}\right)\right]
$$

and

$$
G_{2}(\rho)=\left|\begin{array}{cccc}
1 & 1 & 1 & 1 \\
\mathrm{e}^{\rho} \mathrm{e}^{-\rho} & \mathrm{e}^{\mathrm{i} \rho} & \mathrm{e}^{-\mathrm{i} \rho} \\
\mathrm{e}^{\rho} \mathrm{e}^{-\rho} & -\mathrm{e}^{\mathrm{i} \rho} & -\mathrm{e}^{-\mathrm{i} \rho} \\
1 & 1 & -1 & -1
\end{array}\right|=-2 \mathrm{e}^{\rho}\left[2 \mathrm{e}^{\mathrm{i} \rho}-2 \mathrm{e}^{-\mathrm{i} \rho}+\mathcal{O}\left(\mathrm{e}^{-\rho}\right)\right] .
$$

Hence,

$$
\begin{aligned}
\operatorname{det} \Delta(\rho)= & -2 \rho^{5} \mathrm{e}^{\rho}\left\{\left[\mathrm{e}^{\sqrt{\mathrm{i}} \rho}-\mathrm{e}^{-\sqrt{\mathrm{i}} \rho}\right]\left[(1+i) \mathrm{e}^{\mathrm{i} \rho}+(1-i) \mathrm{e}^{-\mathrm{i} \rho}\right]\right. \\
& \left.+2 \sqrt{i}\left[\mathrm{e}^{\sqrt{\mathrm{i}} \rho}+\mathrm{e}^{-\sqrt{\mathrm{i}} \rho}\right]\left[\mathrm{e}^{\mathrm{i} \rho}-\mathrm{e}^{-\mathrm{i} \rho}\right]+\mathcal{O}\left(\mathrm{e}^{-\rho}\right)\right\} \\
= & -2 \rho^{5} \mathrm{e}^{\rho}\left\{a_{1} \mathrm{e}^{\mathrm{i} \rho} \mathrm{e}^{\sqrt{\mathrm{i}} \rho}+a_{2} \mathrm{e}^{\mathrm{i} \rho} \mathrm{e}^{-\sqrt{\mathrm{i}} \rho}+a_{3} \mathrm{e}^{-\mathrm{i} \rho} \mathrm{e}^{\sqrt{\mathrm{i}} \rho}+a_{4} \mathrm{e}^{-\mathrm{i} \rho} \mathrm{e}^{-\sqrt{\mathrm{i}} \rho}+\mathcal{O}\left(\mathrm{e}^{-\rho}\right)\right\},
\end{aligned}
$$

where $a_{i}, i=1,2,3,4$, are given by (3.11). Moreover, when $\rho \in \mathcal{S}_{1}$ and $\rho \in \mathcal{S}_{2}$, from (3.6), we have

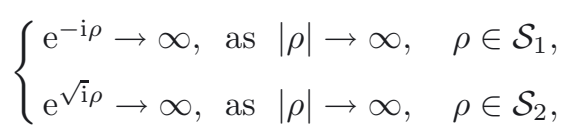

and hence, $\operatorname{det} \Delta(\lambda)$ has more accurate asymptotic expressions given by (3.12) and (3.13) in $\mathcal{S}_{1}$ and $\mathcal{S}_{2}$, respectively.

Theorem 3.3. Let $\mathcal{A}$ be defined by (2.1). The spectrum $\sigma(\mathcal{A})$ has two families:

$$
\sigma(\mathcal{A})=\left\{\lambda_{n}^{p}, n \in \mathbb{N}\right\} \cup\left\{\lambda_{n}^{e}, \overline{\lambda_{n}^{e}}, n \in \mathbb{N}\right\}
$$


where $\lambda_{n}^{p}$ and $\lambda_{n}^{e}$ have the following asymptotic expansions:

$$
\left\{\begin{array}{l}
\lambda_{n}^{p}=-\left[n \pi+\frac{1}{2} \theta_{p}\right]^{2}+\mathcal{O}\left(n \mathrm{e}^{-k_{1} n}\right), \\
\lambda_{n}^{e}=\left[n \pi+\frac{1}{2} \theta_{e}\right] \ln r+\frac{1}{4}\left[\left(2 n \pi+\theta_{e}\right)^{2}-(\ln r)^{2}\right] i+\mathcal{O}\left(n \mathrm{e}^{-k_{2} n}\right),
\end{array}\right.
$$

and

$$
\theta_{p}=\pi-\arctan 2 \sqrt{2}, \quad \theta_{e}=\arctan \frac{\sqrt{2}}{2}, \quad r=\frac{\sqrt{3}}{1+\sqrt{2}}<1, \quad \ln r<0 .
$$

Therefore,

$$
\operatorname{Re} \lambda_{n}^{p}, \operatorname{Re} \lambda_{n}^{e} \rightarrow-\infty, \quad \text { as } n \rightarrow \infty .
$$

Proof. Let $\operatorname{det} \Delta(\rho)=0$. By (3.12), $\rho \in \mathcal{S}_{1}$ satisfies

$$
a_{3} \mathrm{e}^{\sqrt{\mathrm{i}} \rho}+a_{4} \mathrm{e}^{-\sqrt{\mathrm{i}} \rho}+\mathcal{O}\left(\mathrm{e}^{-k_{1}|\rho|}\right)=0 .
$$

By (3.11), $a_{3} \mathrm{e}^{\sqrt{\mathrm{i} \rho}}+a_{4} \mathrm{e}^{-\sqrt{\mathrm{i} \rho}}=0$ yields

$$
\mathrm{e}^{2 \sqrt{i} \rho}=-\frac{a_{4}}{a_{3}}=\frac{1+\sqrt{2}+i(\sqrt{2}-1)}{1-\sqrt{2}-i(1+\sqrt{2})}=\frac{-1+2 \sqrt{2} i}{3}=\mathrm{e}^{\mathrm{i} \theta_{p}}
$$

where $\theta_{p}$ is given by (3.18). Hence, the roots of $a_{3} \mathrm{e}^{\sqrt{\mathrm{i} \rho}}+a_{4} \mathrm{e}^{-\sqrt{\mathrm{i}} \rho}=0$ are

$$
\tilde{\rho}_{n}^{p}=\left[n \pi+\frac{1}{2} \theta_{p}\right] \sqrt{i}, \quad n=0,1,2, \ldots
$$

By Rouché's theorem, the roots of (3.20) have the following asymptotic expression

$$
\rho_{n}^{p}=\left[n \pi+\frac{1}{2} \theta_{p}\right] \sqrt{i}+\mathcal{O}\left(\mathrm{e}^{-k_{1} n}\right), \quad n>N_{1},
$$

where $N_{1}$ is a sufficiently large positive integer. Similarly, from (3.13), it follows that $\rho \in \mathcal{S}_{2}$ satisfies

$$
a_{1} \mathrm{e}^{\mathrm{i} \rho}+a_{3} \mathrm{e}^{-\mathrm{i} \rho}+\mathcal{O}\left(\mathrm{e}^{-k_{2}|\rho|}\right)=0 .
$$

By $(3.11), a_{1} \mathrm{e}^{\mathrm{i} \rho}+a_{3} \mathrm{e}^{-\mathrm{i} \rho}=0$ yields

$$
\mathrm{e}^{2 i \rho}=-\frac{a_{3}}{a_{1}}=-\frac{1-\sqrt{2}-i(1+\sqrt{2})}{1+\sqrt{2}+i(1+\sqrt{2})}=\frac{2+\sqrt{2}+i(1+\sqrt{2})}{3+2 \sqrt{2}}=r \mathrm{e}^{\mathrm{i} \theta_{e}},
$$

where $\theta_{e}$ and $r$ are given by (3.18). Hence, the roots of $a_{1} \mathrm{e}^{\mathrm{i} \rho}+a_{3} \mathrm{e}^{-\mathrm{i} \rho}=0$ are

$$
\tilde{\rho}_{n}^{e}=\frac{1}{2 i}\left[\ln r+\left(2 n \pi+\theta_{e}\right) i\right], \quad n=0,1,2, \ldots
$$

By Rouché's theorem, the roots of (3.23) have the following asymptotic expression

$$
\rho_{n}^{e}=\frac{1}{2 i}\left[\ln r+\left(2 n \pi+\theta_{e}\right) i\right]+\mathcal{O}\left(\mathrm{e}^{-k_{2} n}\right), \quad n>N_{2},
$$

where $N_{2}$ is a sufficiently large positive integer. Finally, by using $\lambda=i \rho^{2}$, we eventually get $\lambda_{n}^{p}$ and $\lambda_{n}^{e}$ given by (3.17).

We now investigate the asymptotic behavior of the eigenfunctions. 
Theorem 3.4. Let $\mathcal{A}$ be defined by (2.1), let $\sigma(\mathcal{A})=\left\{\lambda_{n}^{p}, n \in \mathbb{N}\right\} \cup\left\{\lambda_{n}^{e}, \overline{\lambda_{n}^{e}}, n \in \mathbb{N}\right\}$ be the spectrum of $\mathcal{A}$, and let $\lambda_{n}^{p}:=i\left(\rho_{n}^{p}\right)^{2}$ and $\lambda_{n}^{e}:=i\left(\rho_{n}^{e}\right)^{2}$ with $\rho_{n}^{p}, \rho_{n}^{e}$ given by (3.22) and (3.25), respectively. Then there are two families of approximate normalized eigenfunctions of $\mathcal{A}$ :

(i) One family $\left\{\Phi_{n}^{p}=\left(f_{n}^{p}, \lambda_{n}^{p} f_{n}^{p}, h_{n}^{p}\right), n \in \mathbb{N}\right\}$, where $\Phi_{n}^{p}$ is the eigenfunction of $\mathcal{A}$ with respect to the eigenvalue $\lambda_{n}^{p}$, has the following asymptotic expression:

$$
\left(\begin{array}{c}
\left(f_{n}^{p}\right)^{\prime \prime}(x) \\
\lambda_{n}^{p} f_{n}^{p}(x) \\
h_{n}^{p}(x)
\end{array}\right)=\left(\begin{array}{c}
-2 \sqrt{i}\left[\varphi_{n 1}^{p}(x)+\varphi_{n 2}^{p}(x)\right] \\
2 i \sqrt{i}\left[\varphi_{n 1}^{p}(x)-\varphi_{n 2}^{p}(x)\right] \\
a_{3} \varphi_{n 3}^{p}(x)+a_{4} \varphi_{n 4}^{p}(x)
\end{array}\right)+\mathcal{O}\left(\mathrm{e}^{-k_{1} n}\right),
$$

where $\varphi_{n j}^{p}(x), j=1,2,3,4$, have the following forms:

$$
\left\{\begin{array}{l}
\varphi_{n 1}^{p}(x)=\mathrm{e}^{\mathrm{i} \rho_{n}^{p} x}=\mathrm{e}^{\mathrm{i} \sqrt{i}\left[n \pi+\frac{1}{2} \theta_{p}\right] x+\mathcal{O}\left(\mathrm{e}^{-k_{1} n}\right)}=\mathrm{e}^{\left[-\frac{\sqrt{2}}{2}+i \frac{\sqrt{2}}{2}\right]\left[n \pi+\frac{1}{2} \theta_{p}\right] x+\mathcal{O}\left(\mathrm{e}^{-k_{1} n}\right)}, \\
\varphi_{n 2}^{p}(x)=\mathrm{e}^{-\rho_{n}^{p} x}=\mathrm{e}^{-\sqrt{\mathrm{i}}\left[n \pi+\frac{1}{2} \theta_{p}\right] x+\mathcal{O}\left(\mathrm{e}^{-k_{1} n}\right)}=\mathrm{e}^{\left[-\frac{\sqrt{2}}{2}-i \frac{\sqrt{2}}{2}\right]\left[n \pi+\frac{1}{2} \theta_{p}\right] x+\mathcal{O}\left(\mathrm{e}^{-k_{1} n}\right)}, \\
\varphi_{n 3}^{p}(x)=\mathrm{e}^{\sqrt{\mathrm{i}} \rho_{n}^{p} x}=\mathrm{e}^{\mathrm{i}\left[n \pi+\frac{1}{2} \theta_{p}\right] x+\mathcal{O}\left(\mathrm{e}^{-k_{1} n}\right)}, \\
\varphi_{n 4}^{p}(x)=\mathrm{e}^{-\sqrt{\mathrm{i}} \rho_{n}^{p} x}=\mathrm{e}^{-\mathrm{i}\left[n \pi+\frac{1}{2} \theta_{p}\right] x+\mathcal{O}\left(\mathrm{e}^{-k_{1} n}\right)},
\end{array}\right.
$$

$a_{3}, a_{4}, \theta_{p}$ are constants given by (3.11) and (3.18), respectively, and $\mathcal{O}\left(\mathrm{e}^{-k_{1} n}\right)$ is uniform with respect to $x \in[0,1]$. Furthermore, $\Phi_{n}^{p}=\left(f_{n}^{p}, \lambda_{n}^{p} f_{n}^{p}, h_{n}^{p}\right)$ are approximately normalized in $\mathcal{H}$ in the sense that there exist positive constants $b_{1}$ and $b_{2}$ independent of $n$, such that for all $n$

$$
b_{1} \leq\left\|\Phi_{n}^{p}\right\|=\left\|\left(f_{n}^{p}\right)^{\prime \prime}\right\|_{L^{2}(0,1)}+\left\|\lambda_{n}^{p} f_{n}^{p}\right\|_{L^{2}(0,1)}+\left\|h_{n}^{p}\right\|_{L^{2}(0,1)} \leq b_{2} .
$$

(ii) The other family $\left\{\Phi_{n}^{e}=\left(f_{n}^{e}, \lambda_{n}^{e} f_{n}^{e}, h_{n}^{e}\right), \overline{\Phi_{n}^{e}}=\left(\overline{f_{n}^{e}}, \overline{\lambda_{n}^{e} f_{n}^{e}}, \bar{h}_{2 n}\right), n \in \mathbb{N}\right\}$, where $\Phi_{n}^{e}$ and $\overline{\Phi_{n}^{e}}$ are the eigenfunctions of $\mathcal{A}$ with respect to the complex conjugate eigenvalue pairs $\lambda_{n}^{e}$ and $\overline{\lambda_{n}^{e}}$, respectively, has the following asymptotic expression:

$$
\left(\begin{array}{c}
\left(f_{n}^{e}\right)^{\prime \prime}(x) \\
\lambda_{n}^{e} f_{n}^{e}(x) \\
h_{n}^{e}(x)
\end{array}\right)=\left(\begin{array}{c}
\varphi_{n 1}^{e}(x)-\varphi_{n 2}^{e}(x)+2 \sinh \left[\frac{1}{2} \ln r+\left(n \pi+\frac{1}{2} \theta_{e}\right) i\right] \varphi_{n 3}^{e}(x) \\
i \varphi_{n 2}^{e}(x)-i \varphi_{n 1}^{e}(x)+2 i \sinh \left[\frac{1}{2} \ln r+\left(n \pi+\frac{1}{2} \theta_{e}\right) i\right] \varphi_{n 3}^{e}(x) \\
4 \sinh \left[\frac{1}{2} \ln r+\left(n \pi+\frac{1}{2} \theta_{e}\right) i\right] \varphi_{n 4}^{e}(x)
\end{array}\right)+\mathcal{O}\left(\mathrm{e}^{-k_{2} n}\right),
$$

where $\varphi_{n j}^{e}(x), j=1,2,3,4$, are given by

$$
\left\{\begin{array}{l}
\varphi_{n 1}^{e}(x)=\mathrm{e}^{\mathrm{i} \rho_{n}^{e}(1-x)}=\mathrm{e}^{\frac{1}{2}\left[\ln r+\left(2 n \pi+\theta_{e}\right) i\right](1-x)+\mathcal{O}\left(\mathrm{e}^{-k_{2} n}\right)}, \\
\varphi_{n 2}^{e}(x)=\mathrm{e}^{-\mathrm{i} \rho_{n}^{e}(1-x)}=\mathrm{e}^{-\frac{1}{2}\left[\ln r+\left(2 n \pi+\theta_{e}\right) i\right](1-x)+\mathcal{O}\left(\mathrm{e}^{-k_{2} n}\right)}, \\
\varphi_{n 3}^{e}(x)=\mathrm{e}^{-\rho_{n}^{e} x}=\mathrm{e}^{\frac{1}{2}\left[i \ln r-\left(2 n \pi+\theta_{e}\right)\right] x+\mathcal{O}\left(\mathrm{e}^{-k_{2} n}\right)}, \\
\varphi_{n 4}^{e}(x)=\mathrm{e}^{-\sqrt{\mathrm{i}} \rho_{n}^{e} x}=\mathrm{e}^{\frac{1}{2} \sqrt{i}\left[i \ln r-\left(2 n \pi+\theta_{e}\right)\right] x+\mathcal{O}\left(\mathrm{e}^{-k_{2} n}\right)},
\end{array}\right.
$$

$\theta_{e}, r$ are constants given by (3.18), and $\mathcal{O}\left(\mathrm{e}^{-k_{2} n}\right)$ is uniform with respect to $x \in[0,1]$. Furthermore, $\Phi_{n}^{e}=\left(f_{n}^{e}, \lambda_{n}^{e} f_{n}^{e}, h_{n}^{e}\right)$ are approximately normalized in $\mathcal{H}$ in the sense that there exist positive constants $b_{3}$ and $b_{4}$ independent of $n$, such that for all $n$

$$
b_{3} \leq\left\|\Phi_{n}^{e}\right\|=\left\|f_{2 n}^{\prime \prime}\right\|_{L^{2}(0,1)}+\left\|\lambda_{n}^{e} f_{n}^{e}\right\|_{L^{2}(0,1)}+\left\|h_{n}^{e}\right\|_{L^{2}(0,1)} \leq b_{4} .
$$


Proof. First we look for $\Phi_{n}^{p}$ of $\mathcal{A}$ with respect to $\lambda_{n}^{p}$. From (3.4)-(3.9), and some linear algebra calculations, for $\rho \in \mathcal{S}_{1}, h^{p}(x)$ is given by

$$
\begin{aligned}
h^{p}(x) & =\left|\begin{array}{cccccc}
1 & 1 & 1 & 1 & 0 & 0 \\
\mathrm{e}^{\rho} & \mathrm{e}^{-\rho} & \mathrm{e}^{\mathrm{i} \rho} & \mathrm{e}^{-\mathrm{i} \rho} & 0 & 0 \\
\rho^{2} \mathrm{e}^{\rho} & \rho^{2} \mathrm{e}^{-\rho} & -\rho^{2} \mathrm{e}^{\mathrm{i} \rho} & -\rho^{2} \mathrm{e}^{-\mathrm{i} \rho} & 0 & 0 \\
0 & 0 & 0 & 0 & \mathrm{e}^{\sqrt{\mathrm{i}} \rho x} & \mathrm{e}^{-\sqrt{\mathrm{i}} \rho x} \\
\rho^{2} & \rho^{2} & -\rho^{2} & -\rho^{2} & -1 & -1 \\
i \rho^{3} & -i \rho^{3} & -\rho^{3} & \rho^{3} & \sqrt{i} \rho & -\sqrt{\mathrm{i} \rho}
\end{array}\right| \\
& =-\rho^{5}\left[\mathrm{e}^{\sqrt{\mathrm{i}} \rho x}-\mathrm{e}^{-\sqrt{\mathrm{i}} \rho x}\right] G_{1}(\rho)+\sqrt{i} \rho^{5}\left[\mathrm{e}^{\sqrt{\mathrm{i}} \rho x}+\mathrm{e}^{-\sqrt{\mathrm{i}} \rho x}\right] G_{2}(\rho),
\end{aligned}
$$

where $G_{1}(\rho)$ and $G_{2}(\rho)$ are given by (3.14) and (3.15), respectively. Hence,

$$
h^{p}(x)=-2 \rho^{5} \mathrm{e}^{\rho}\left\{a_{1} \mathrm{e}^{\mathrm{i} \rho} \mathrm{e}^{\sqrt{\mathrm{i}} \rho x}+a_{2} \mathrm{e}^{\mathrm{i} \rho} \mathrm{e}^{-\sqrt{\mathrm{i}} \rho x}+a_{3} \mathrm{e}^{-\mathrm{i} \rho} \mathrm{e}^{\sqrt{\mathrm{i} \rho} \rho}+a_{4} \mathrm{e}^{-\mathrm{i} \rho} \mathrm{e}^{-\sqrt{\mathrm{i}} \rho x}+\mathcal{O}\left(\mathrm{e}^{-\rho}\right)\right\},
$$

where $a_{i}, i=1,2,3,4$, are given by $(3.11)$ and $\mathcal{O}\left(\mathrm{e}^{-\rho}\right)$ is uniform with respect to $x \in[0,1]$. Since $\rho \in \mathcal{S}_{1}$, we have $\mathrm{e}^{-\mathrm{i} \rho} \rightarrow \infty$, as $|\rho| \rightarrow \infty$, and hence,

$$
h^{p}(x)=-2 \rho^{5} \mathrm{e}^{\rho} \mathrm{e}^{-\mathrm{i} \rho}\left\{a_{3} \mathrm{e}^{\sqrt{\mathrm{i}} \rho x}+a_{4} \mathrm{e}^{-\sqrt{\mathrm{i}} \rho x}+\mathcal{O}\left(\mathrm{e}^{-k_{1}|\rho|}\right)\right\}
$$

where $\mathcal{O}\left(\mathrm{e}^{-k_{1}|\rho|}\right)$ is uniform with respect to $x \in[0,1]$. Similarly,

$$
f^{p}(x)=\left|\begin{array}{cccccc}
1 & 1 & 1 & 1 & 0 & 0 \\
\mathrm{e}^{\rho} & \mathrm{e}^{-\rho} & \mathrm{e}^{\mathrm{i} \rho} & \mathrm{e}^{-\mathrm{i} \rho} & 0 & 0 \\
\rho^{2} \mathrm{e}^{\rho} & \rho^{2} \mathrm{e}^{-\rho} & -\rho^{2} \mathrm{e}^{\mathrm{i} \rho} & -\rho^{2} \mathrm{e}^{-\mathrm{i} \rho} & 0 & 0 \\
\mathrm{e}^{\rho x} & \mathrm{e}^{-\rho x} & \mathrm{e}^{\mathrm{i} \rho x} & \mathrm{e}^{-\mathrm{i} \rho x} & 0 & 0 \\
\rho^{2} & \rho^{2} & -\rho^{2} & -\rho^{2} & -1 & -1 \\
i \rho^{3} & -i \rho^{3} & -\rho^{3} & \rho^{3} & \sqrt{i} \rho-\sqrt{\mathrm{i} \rho}
\end{array}\right|=-4 \sqrt{i} \rho^{3} \mathrm{e}^{\rho} \mathrm{e}^{-\mathrm{i} \rho}\left[\mathrm{e}^{\mathrm{i} \rho x}-\mathrm{e}^{-\rho x}+\mathcal{O}\left(\mathrm{e}^{-k_{1}|\rho|}\right)\right]
$$

and

$$
\left(f^{p}\right)^{\prime \prime}(x)=4 \sqrt{i} \rho^{5} \mathrm{e}^{\rho} \mathrm{e}^{-\mathrm{i} \rho}\left[\mathrm{e}^{\mathrm{i} \rho x}+\mathrm{e}^{-\rho x}+\mathcal{O}\left(\mathrm{e}^{-k_{1}|\rho|}\right)\right] .
$$

By setting

$$
\Phi_{n}^{p}=\left(\begin{array}{c}
f_{n}^{p}(x) \\
\lambda_{n}^{p} f_{n}^{p}(x) \\
h_{n}^{p}(x)
\end{array}\right)=-\frac{1}{2}\left(\rho_{n}^{p}\right)^{-5} \mathrm{e}^{-\rho_{n}^{p}} \mathrm{e}^{\mathrm{i} \rho_{n}^{p}}\left(\begin{array}{c}
f^{p}\left(x, \rho_{n}^{p}\right) \\
i\left(\rho_{n}^{p}\right)^{2} f^{p}\left(x, \rho_{n}^{p}\right) \\
h^{p}\left(x, \rho_{n}^{p}\right)
\end{array}\right)
$$

we get

$$
\left(\begin{array}{c}
\left(f_{n}^{p}\right)^{\prime \prime}(x) \\
\lambda_{n}^{p} f_{n}^{p}(x) \\
h_{n}^{p}(x)
\end{array}\right)=\left(\begin{array}{c}
-2 \sqrt{i} \mathrm{e}^{\mathrm{i} \rho_{n}^{p} x}-2 \sqrt{i} \mathrm{e}^{-\rho_{n}^{p} x} \\
2 i \sqrt{i} \mathrm{e}^{\mathrm{i} \rho_{n}^{p} x}-2 i \sqrt{i} \mathrm{e}^{-\rho_{n}^{p} x} \\
a_{3} \mathrm{e}^{\sqrt{\mathrm{i}} \rho_{n}^{p} x}+a_{4} \mathrm{e}^{-\sqrt{\mathrm{i}} \rho_{n}^{p} x}
\end{array}\right)+\mathcal{O}\left(\mathrm{e}^{-k_{1}\left|\rho_{n}^{p}\right|}\right),
$$


where $a_{3}, a_{4}$ are given by $(3.11)$ and $\mathcal{O}\left(\mathrm{e}^{-k_{1}\left|\rho_{n}^{p}\right|}\right)$ is uniform with respect to $x \in[0,1]$. Substituting $\rho_{n}^{p}$ given by (3.22) into (3.32) yield (3.26). Noting that from (3.27), we have

$$
\left\{\begin{array}{l}
\left\|\varphi_{n 1}^{p}\right\|_{L^{2}(0,1)}^{2}=\mathcal{O}\left(n^{-1}\right), \quad\left\|\varphi_{n 2}^{p}\right\|_{L^{2}(0,1)}^{2}=\mathcal{O}\left(n^{-1}\right) \\
\left\|\varphi_{n 3}^{p}\right\|_{L^{2}(0,1)}^{2}=1+\mathcal{O}\left(\mathrm{e}^{-k_{1} n}\right), \quad\left\|\varphi_{n 4}^{p}\right\|_{L^{2}(0,1)}^{2}=1+\mathcal{O}\left(\mathrm{e}^{-k_{1} n}\right) \\
\left\|a_{3} \varphi_{n 3}^{p}+a_{4} \varphi_{n 4}^{p}\right\|_{L^{2}(0,1)}^{2}=\left|a_{3}\right|^{2}+\left|a_{4}\right|^{2}+\mathcal{O}\left(n^{-1}\right)
\end{array}\right.
$$

These together (3.26), (3.27) yield (3.28). Now we are going to look for $\Phi_{n}^{e}$. For $\rho \in \mathcal{S}_{2}$, we have $\mathrm{e}^{\sqrt{\mathrm{i}} \rho} \rightarrow \infty$, as $|\rho| \rightarrow \infty$. Similarly, we get

$$
\begin{aligned}
& h^{e}(x)=\left|\begin{array}{cccccc}
1 & 1 & 1 & 1 & 0 & 0 \\
\mathrm{e}^{\rho} & \mathrm{e}^{-\rho} & \mathrm{e}^{\mathrm{i} \rho} & \mathrm{e}^{-\mathrm{i} \rho} & 0 & 0 \\
\rho^{2} \mathrm{e}^{\rho} & \rho^{2} \mathrm{e}^{-\rho} & -\rho^{2} \mathrm{e}^{\mathrm{i} \rho} & -\rho^{2} \mathrm{e}^{-\mathrm{i} \rho} & 0 & 0 \\
0 & 0 & 0 & 0 & \mathrm{e}^{\sqrt{\mathrm{i}} \rho} & \mathrm{e}^{-\sqrt{\mathrm{i}} \rho} \\
\rho^{2} & \rho^{2} & -\rho^{2} & -\rho^{2} & -1 & -1 \\
0 & 0 & 0 & 0 & \mathrm{e}^{\sqrt{\mathrm{i}} \rho x} & \mathrm{e}^{-\sqrt{\mathrm{i}} \rho x}
\end{array}\right| \\
& =8 i \rho^{4} \mathrm{e}^{\rho}\left[\mathrm{e}^{\sqrt{\mathrm{i}} \rho} \mathrm{e}^{-\sqrt{\mathrm{i}} \rho x}-\mathrm{e}^{-\sqrt{\mathrm{i}} \rho(1-x)}\right]\left[\sin \rho+\mathcal{O}\left(\mathrm{e}^{-\rho}\right)\right], \\
& \begin{aligned}
f^{e}(x) & =\left|\begin{array}{cccccc}
1 & 1 & 1 & 1 & 0 & 0 \\
\mathrm{e}^{\rho} & \mathrm{e}^{-\rho} & \mathrm{e}^{\mathrm{i} \rho} & \mathrm{e}^{-\mathrm{i} \rho} & 0 & 0 \\
\rho^{2} \mathrm{e}^{\rho} \rho^{2} \mathrm{e}^{-\rho} & -\rho^{2} \mathrm{e}^{\mathrm{i} \rho} & -\rho^{2} \mathrm{e}^{-\mathrm{i} \rho} & 0 & 0 \\
0 & 0 & 0 & 0 & \mathrm{e}^{\sqrt{\mathrm{i}} \rho} & \mathrm{e}^{-\sqrt{\mathrm{i}} \rho} \\
\rho^{2} & \rho^{2} & -\rho^{2} & -\rho^{2} & -1 & -1 \\
\mathrm{e}^{\rho x} & \mathrm{e}^{-\rho x} & \mathrm{e}^{\mathrm{i} \rho x} & \mathrm{e}^{-\mathrm{i} \rho x} & 0 & 0
\end{array}\right| \\
& =-2 \rho^{2} \mathrm{e}^{\rho}\left[\mathrm{e}^{\sqrt{\mathrm{i}} \rho}-\mathrm{e}^{-\sqrt{\mathrm{i}} \rho}\right]\left[\mathrm{e}^{\mathrm{i} \rho(1-x)}-\mathrm{e}^{-\mathrm{i} \rho(1-x)}-2 i \sin \rho \mathrm{e}^{-\rho x}+\mathcal{O}\left(\mathrm{e}^{-|\rho|}\right)\right]
\end{aligned}
\end{aligned}
$$

and

$$
\left(f^{e}\right)^{\prime \prime}(x)=2 \rho^{4} \mathrm{e}^{\rho}\left[\mathrm{e}^{\sqrt{\mathrm{i}} \rho}-\mathrm{e}^{-\sqrt{\mathrm{i}} \rho}\right]\left[\mathrm{e}^{\mathrm{i} \rho(1-x)}-\mathrm{e}^{-\mathrm{i} \rho(1-x)}+2 i \sin \rho \mathrm{e}^{-\rho x}+\mathcal{O}\left(\mathrm{e}^{-|\rho|}\right)\right] .
$$

By setting

$$
\Phi_{n}^{e}=\left(\begin{array}{c}
f_{n}^{e}(x) \\
\lambda_{n}^{e} f_{n}^{e}(x) \\
h_{n}^{e}(x)
\end{array}\right)=\frac{1}{2}\left(\rho_{n}^{e}\right)^{-4} \mathrm{e}^{-\rho_{n}^{e}} \mathrm{e}^{-\sqrt{\mathrm{i}} \rho_{n}^{e}}\left(\begin{array}{c}
f^{e}\left(x, \rho_{n}^{e}\right) \\
i\left(\rho_{n}^{e}\right)^{2} f^{e}\left(x, \rho_{n}^{e}\right) \\
h^{e}\left(x, \rho_{n}^{e}\right)
\end{array}\right)
$$

we get

$$
\left(\begin{array}{c}
\left(f_{n}^{e}\right)^{\prime \prime}(x) \\
\lambda_{n}^{e} f_{n}^{e}(x) \\
h_{n}^{e}(x)
\end{array}\right)=\left(\begin{array}{c}
\mathrm{e}^{\mathrm{i} \rho_{n}^{e}(1-x)}-\mathrm{e}^{-\mathrm{i} \rho_{n}^{e}(1-x)}+2 i \sin \rho_{n}^{e} \mathrm{e}^{-\rho_{n}^{e} x} \\
-i \mathrm{e}^{\mathrm{i} \rho_{n}^{e}(1-x)}+i \mathrm{e}^{-\mathrm{i} \rho_{n}^{e}(1-x)}-2 \sin \rho_{n}^{e} \mathrm{e}^{-\rho_{n}^{e} x} \\
4 i \sin \rho_{n}^{e} \mathrm{e}^{-\sqrt{\mathrm{i}} \rho_{n}^{e} x}
\end{array}\right)+\mathcal{O}\left(\mathrm{e}^{-k_{2}\left|\rho_{n}^{e}\right|}\right)
$$


where $\mathcal{O}\left(\mathrm{e}^{-k_{2}\left|\rho_{n}^{e}\right|}\right)$ is uniform with respect to $x \in[0,1]$. Substituting $\rho_{n}^{e}$ given by (3.25) into the above equation and nothing that $\sin \rho_{n}^{e}=-i \sinh \left[\frac{1}{2} \ln r+\left(n \pi+\frac{1}{2} \theta_{e}\right) i\right]+\mathcal{O}\left(\mathrm{e}^{-k_{2} n}\right)$, we get (3.29). Noting from (3.30), we have

$$
\left\{\begin{array}{l}
\left\|\varphi_{n 1}^{e}\right\|_{L^{2}(0,1)}^{2}=[r-1][\ln r]^{-1}+\mathcal{O}\left(\mathrm{e}^{-k_{2} n}\right) \\
\left\|\varphi_{n 2}^{e}\right\|_{L^{2}(0,1)}^{2}=\left[1-\frac{1}{r}\right][\ln r]^{-1}+\mathcal{O}\left(\mathrm{e}^{-k_{2} n}\right), \\
\left\|\varphi_{n 3}^{e}\right\|_{L^{2}(0,1)}^{2}=\mathcal{O}\left(n^{-1}\right), \quad\left\|\varphi_{n 4}^{e}\right\|_{L^{2}(0,1)}^{2}=\mathcal{O}\left(n^{-1}\right) \\
\left\|\varphi_{n 1}^{e}-\varphi_{n 2}^{e}\right\|_{L^{2}(0,1)}^{2}=\left[r-\frac{1}{r}\right][\ln r]^{-1}+\mathcal{O}\left(n^{-1}\right)
\end{array}\right.
$$

These together with (3.29), (3.30) yield (3.31). The proof is complete.

To end this section, we remark that the same process can be used to produce asymptotic expansions for the eigenpairs of $\mathcal{A}^{*}$, the adjoint operator of $\mathcal{A}$,

$$
\left\{\begin{array}{l}
\mathcal{A}^{*}(f, g, h)=\left(-g, f^{(4)}, h^{\prime \prime}\right), \forall(f, g, h) \in D(\mathcal{A}) \\
D\left(\mathcal{A}^{*}\right)=\left\{(f, g, h) \in\left(H^{4} \times H_{L}^{2} \times H^{2}\right) \cap \mathcal{H} \mid \begin{array}{l}
h(1)=f^{\prime \prime}(1)=0 \\
g^{\prime}(0)=h^{\prime}(0), f^{\prime \prime}(0)=h(0)
\end{array}\right\} .
\end{array}\right.
$$

It is because when $\mathcal{A}$ is a discrete operator, so is $\mathcal{A}^{*}$ ([2], p. 2354); and when the eigenvalues of $\mathcal{A}$ are symmetric about the real axis, then $\mathcal{A}^{*}$ will have the same eigenvalues as $\mathcal{A}([10]$, p. 26) with the same algebraic multiplicity for the conjugate eigenvalues ([2], p. 2354 or [3], p. 10). Moreover, we can get the asymptotic eigenfunctions of $\mathcal{A}^{*}$. Actually, from $\mathcal{A}^{*} X=\lambda X$, where $X=(f, g, h)$ is the eigenfunction of $\mathcal{A}^{*}$ with respect to the eigenvalue $\lambda$, we have that $g=-\lambda f$ and that $f, h$ satisfy the following equation:

$$
\left\{\begin{array}{l}
f^{(4)}(x)+\lambda^{2} f(x)=0 \\
h^{\prime \prime}(x)-\lambda h(x)=0 \\
f(0)=f(1)=f^{\prime \prime}(1)=h(1)=0 \\
f^{\prime \prime}(0)=h(0), \lambda f^{\prime}(0)=-h^{\prime}(0)
\end{array}\right.
$$

This problem is the same as (3.1). So the eigenfunctions of $\mathcal{A}^{*}$ are obtained as in the proof of Theorem 3.4.

Theorem 3.5. Let $\mathcal{A}^{*}$ be defined by (3.34), let $\sigma\left(\mathcal{A}^{*}\right)=\sigma(\mathcal{A})=\left\{\lambda_{n}^{p}, n \in \mathbb{N}\right\} \cup\left\{\lambda_{n}^{e}, \overline{\lambda_{n}^{e}}, n \in \mathbb{N}\right\}$, and let $\lambda_{n}^{p}:=i\left(\rho_{n}^{p}\right)^{2}$ and $\lambda_{n}^{e}:=i\left(\rho_{n}^{e}\right)^{2}$ with $\rho_{n}^{p}, \rho_{n}^{e}$ given by (3.22) and (3.25), respectively. Then there are two families of approximate normalized eigenfunctions of $\mathcal{A}^{*}$ :

(i) One family $\left\{\Psi_{n}^{p}=\left(f_{n}^{p},-\lambda_{n}^{p} f_{n}^{p}, h_{n}^{p}\right), n \in \mathbb{N}\right\}$, where $\Psi_{n}^{p}$ is the eigenfunction of $\mathcal{A}^{*}$ with respect to the eigenvalue $\lambda_{n}^{p}$, has the following asymptotic expression:

$$
\left(\begin{array}{c}
\left(f_{n}^{p}\right)^{\prime \prime}(x) \\
-\lambda_{n}^{p} f_{n}^{p}(x) \\
h_{n}^{p}(x)
\end{array}\right)=\left(\begin{array}{c}
-2 \sqrt{i}\left[\varphi_{n 1}^{p}(x)+\varphi_{n 2}^{p}(x)\right] \\
2 i \sqrt{i}\left[\varphi_{n 2}^{p}(x)-\varphi_{n 1}^{p}(x)\right] \\
a_{3} \varphi_{n 3}^{p}(x)+a_{4} \varphi_{n 4}^{p}(x)
\end{array}\right)+\mathcal{O}\left(\mathrm{e}^{-k_{1} n}\right)
$$

where $\varphi_{n j}^{p}(x), j=1,2,3,4$, are given by (3.27), $a_{3}, a_{4}$ are constants given by $(3.11)$, and $\mathcal{O}\left(\mathrm{e}^{-k_{1} n}\right)$ is uniform with respect to $x \in[0,1]$. Moreover, $\Psi_{n}^{p}=\left(f_{n}^{p},-\lambda_{n}^{p} f_{n}^{p}, h_{n}^{p}\right)$ are approximately normalized in $\mathcal{H}$. 
(ii) The other family $\left\{\Psi_{n}^{e}=\left(f_{n}^{e},-\lambda_{n}^{e} f_{n}^{e}, h_{n}^{e}\right), \overline{\Psi_{n}^{e}}=\left(\overline{f_{n}^{e}},-\overline{\lambda_{n}^{e} f_{n}^{e}}, \overline{h_{n}^{e}}\right), n \in \mathbb{N}\right\}$, where $\Psi_{2 n}$ and $\bar{\Psi}_{2 n}$ are the eigenfunctions of $\mathcal{A}^{*}$ with respect to the complex conjugate eigenvalue pairs $\lambda_{n}^{e}$ and $\overline{\lambda_{n}^{e}}$, respectively, has the following asymptotic expression:

$$
\left(\begin{array}{c}
\left(f_{n}^{e}\right)^{\prime \prime}(x) \\
-\lambda_{n}^{e} f_{n}^{e}(x) \\
h_{n}^{e}(x)
\end{array}\right)=\left(\begin{array}{c}
\varphi_{n 1}^{e}(x)-\varphi_{n 2}^{e}(x)+2 \sinh \left[\frac{1}{2} \ln r+\left(n \pi+\frac{1}{2} \theta_{e}\right) i\right] \varphi_{n 3}^{e}(x) \\
i \varphi_{n 1}^{e}(x)-i \varphi_{n 2}^{e}(x)-2 i \sinh \left[\frac{1}{2} \ln r+\left(n \pi+\frac{1}{2} \theta_{e}\right) i\right] \varphi_{n 3}^{e}(x) \\
4 \sinh \left[\frac{1}{2} \ln r+\left(n \pi+\frac{1}{2} \theta_{e}\right) i\right] \varphi_{n 4}^{e}(x)
\end{array}\right)+\mathcal{O}\left(\mathrm{e}^{-k_{2} n}\right)
$$

where $\varphi_{n j}^{e}(x), j=1,2,3,4$, are given by (3.30), $\theta_{e}, r$ are constants given by (3.18), and $\mathcal{O}\left(\mathrm{e}^{-k_{2} n}\right)$ is uniform with respect to $x \in[0,1]$. Moreover, $\Psi_{2 n}=\left(f_{n}^{e},-\lambda_{n}^{e} f_{n}^{e}, h_{n}^{e}\right)$ are approximately normalized in $\mathcal{H}$.

\section{Completeness of the Root subspace of the System}

In this section, we are going to show the completeness of the root subspace of the system (2.2).

Lemma 4.1. Let $\mathcal{A}$ be defined by (2.1) and for $x \in[0,1]$ and $\rho \in \mathbb{C}$, let

$$
\left\{\begin{array}{l}
Q_{1}(x, \xi)=\frac{1}{8} \operatorname{sign}(x-\xi) \rho^{-3}\left[\mathrm{e}^{\rho(x-\xi)}-\mathrm{e}^{-\rho(x-\xi)}+i \mathrm{e}^{\mathrm{i} \rho(x-\xi)}-i \mathrm{e}^{-\mathrm{i} \rho(x-\xi)}\right], \\
Q_{2}(x, \xi)=-i \frac{1}{4} \operatorname{sign}(x-\xi) \rho^{-1}\left[\sqrt{i} \mathrm{e}^{\sqrt{\mathrm{i}} \rho(x-\xi)}-\sqrt{\mathrm{i}} \mathrm{e}^{-\sqrt{\mathrm{i}} \rho(x-\xi)}\right] .
\end{array}\right.
$$

For any $\lambda=i \rho^{2} \in \rho(\mathcal{A})$ with $\lambda \neq 0$ and $(\phi, \psi, \chi) \in \mathcal{H}$, let $R(\lambda, \mathcal{A})=(\lambda-\mathcal{A})^{-1}$ be the resolvent operator of $\mathcal{A}$ and let

$$
F_{0}(x, \rho)=\int_{0}^{1} Q_{1}(x, \xi)\left[i \rho^{2} \phi(\xi)+\psi(\xi)\right] \mathrm{d} \xi, \quad H_{0}(x, \rho)=-\int_{0}^{1} Q_{2}(x, \xi) \chi(\xi) \mathrm{d} \xi .
$$

Then the solution of the resolvent equation $R(\lambda, \mathcal{A})(\phi, \psi, \chi)=(f, g, h)$ is given by

$$
f(x)=\frac{F(x, \rho)}{\operatorname{det} \Delta(\rho)}, \quad g(x)=\lambda f(x)-\phi(x), \quad h(x)=\frac{H(x, \rho)}{\operatorname{det} \Delta(\rho)},
$$

where $\Delta(\rho)$ is defined by (3.9),

$$
\begin{gathered}
F(x, \rho)=\left|\begin{array}{ccccccc}
\mathrm{e}^{\rho x} & \mathrm{e}^{-\rho x} & \mathrm{e}^{\mathrm{i} \rho x} & \mathrm{e}^{-\mathrm{i} \rho x} & 0 & 0 & F_{0}(x, \rho) \\
1 & 1 & 1 & 1 & 0 & 0 & F_{1} \\
\mathrm{e}^{\rho} & \mathrm{e}^{-\rho} & \mathrm{e}^{\mathrm{i} \rho} & \mathrm{e}^{-\mathrm{i} \rho} & 0 & 0 & F_{2} \\
\rho^{2} \mathrm{e}^{\rho} & \rho^{2} \mathrm{e}^{-\rho} & -\rho^{2} \mathrm{e}^{\mathrm{i} \rho} & -\rho^{2} \mathrm{e}^{-\mathrm{i} \rho} & 0 & 0 & F_{3} \\
0 & 0 & 0 & 0 & \mathrm{e}^{\sqrt{\mathrm{i}} \rho} & \mathrm{e}^{-\sqrt{\mathrm{i}} \rho} & H_{4} \\
\rho^{2} & \rho^{2} & -\rho^{2} & -\rho^{2} & -1 & -1 & F_{5}-H_{5} \\
i \rho^{3} & -i \rho^{3} & -\rho^{3} & \rho^{3} & \sqrt{i} \rho-\sqrt{\mathrm{i} \rho} F_{6}+H_{6}-\phi^{\prime}(0)
\end{array}\right|, \\
H(x, \rho)=\left|\begin{array}{ccccccc}
0 & 0 & 0 & 0 & \mathrm{e}^{\sqrt{\mathrm{i}} \rho x} & \mathrm{e}^{-\sqrt{\mathrm{i}} \rho x} & H_{0}(x, \rho) \\
1 & 1 & 1 & 1 & 0 & 0 & F_{1} \\
\mathrm{e}^{\rho} & \mathrm{e}^{-\rho} & \mathrm{e}^{\mathrm{i} \rho} & \mathrm{e}^{-\mathrm{i} \rho} & 0 & 0 & F_{2} \\
\rho^{2} \mathrm{e}^{\rho} \rho^{2} \mathrm{e}^{-\rho}-\rho^{2} \mathrm{e}^{\mathrm{i} \rho}-\rho^{2} \mathrm{e}^{-\mathrm{i} \rho} & 0 & 0 & F_{3} \\
0 & 0 & 0 & 0 & \mathrm{e}^{\sqrt{\mathrm{i}} \rho} & \mathrm{e}^{-\sqrt{\mathrm{i}} \rho} & H_{4} \\
\rho^{2} & \rho^{2} & -\rho^{2} & -\rho^{2} & -1 & -1 & F_{5}-H_{5} \\
i \rho^{3} & -i \rho^{3} & -\rho^{3} & \rho^{3} & \sqrt{i} \rho & -\sqrt{\mathrm{i} \rho} & F_{6}+H_{6}-\phi^{\prime}(0)
\end{array}\right| .
\end{gathered}
$$


and $F_{j}, j=1,2,3,5,6, H_{s}, s=4,5,6$ are constants given by

$$
\left\{\begin{array}{l}
F_{1}=-\frac{1}{8} \rho^{-3} \int_{0}^{1}\left[\mathrm{e}^{-\rho \xi}-\mathrm{e}^{\rho \xi}+i \mathrm{e}^{-\mathrm{i} \rho \xi}-i \mathrm{e}^{\mathrm{i} \rho \xi}\right]\left[i \rho^{2} \phi(\xi)+\psi(\xi)\right] \mathrm{d} \xi, \\
F_{2}=\frac{1}{8} \rho^{-3} \int_{0}^{1}\left[\mathrm{e}^{\rho(1-\xi)}-\mathrm{e}^{-\rho(1-\xi)}+i \mathrm{e}^{\mathrm{i} \rho(1-\xi)}-i \mathrm{e}^{-\mathrm{i} \rho(1-\xi)}\right]\left[i \rho^{2} \phi(\xi)+\psi(\xi)\right] \mathrm{d} \xi, \\
F_{3}=\frac{1}{8} \rho^{-1} \int_{0}^{1}\left[\mathrm{e}^{\rho(1-\xi)}-\mathrm{e}^{-\rho(1-\xi)}-i \mathrm{e}^{\mathrm{i} \rho(1-\xi)}+i \mathrm{e}^{-\mathrm{i} \rho(1-\xi)}\right]\left[i \rho^{2} \phi(\xi)+\psi(\xi)\right] \mathrm{d} \xi, \\
F_{5}=-\frac{1}{8} \rho^{-1} \int_{0}^{1}\left[\mathrm{e}^{-\rho \xi}-\mathrm{e}^{\rho \xi}-i \mathrm{e}^{-\mathrm{i} \rho \xi}+i \mathrm{e}^{\mathrm{i} \rho \xi}\right]\left[i \rho^{2} \phi(\xi)+\psi(\xi)\right] \mathrm{d} \xi, \\
F_{6}=-i \frac{1}{8} \int_{0}^{1}\left[\mathrm{e}^{-\rho \xi}+\mathrm{e}^{\rho \xi}-\mathrm{e}^{-\mathrm{i} \rho \xi}-\mathrm{e}^{\mathrm{i} \rho \xi}\right]\left[i \rho^{2} \phi(\xi)+\psi(\xi)\right] \mathrm{d} \xi, \\
H_{4}=i \frac{1}{4} \rho^{-1} \int_{0}^{1}\left[\sqrt{i} \mathrm{e}^{\sqrt{\mathrm{i}} \rho(1-\xi)}-\sqrt{\mathrm{i}} \mathrm{e}^{-\sqrt{\mathrm{i}} \rho(1-\xi)}\right] \chi(\xi) \mathrm{d} \xi, \\
H_{6}=\frac{1}{4} \int_{0}^{-1}\left[\mathrm{e}^{-\sqrt{\mathrm{i}} \rho \xi}+\mathrm{e}^{\sqrt{\mathrm{i}} \rho \xi}\right] \chi(\xi) \mathrm{d} \xi .
\end{array}\right.
$$

Proof. For any $(\phi, \psi, \chi) \in \mathcal{H}$ and any $\lambda=i \rho^{2} \in \rho(\mathcal{A})$ with $\rho \neq 0$, solving the resolvent equation

$$
(\lambda-\mathcal{A})(f, g, h)=(\phi, \psi, \chi)
$$

yields $g=\lambda f-\phi$ with $f, h$ satisfying

$$
\left\{\begin{array}{l}
f^{(4)}(x)-\rho^{4} f(x)=i \rho^{2} \phi(x)+\psi(x), \\
h^{\prime \prime}(x)-i \rho^{2} h(x)=-\chi(x), \\
f(0)=f(1)=f^{\prime \prime}(1)=h(1)=0, \\
f^{\prime \prime}(0)=h(0), \\
\lambda f^{\prime}(0)+h^{\prime}(0)=\phi^{\prime}(0) .
\end{array}\right.
$$

Note that $f^{(4)}(x)-\rho^{4} f(x)=i \rho^{2} \phi(x)+\psi(x)$ and $h^{\prime \prime}(x)-i \rho^{2} h(x)=-\chi(x)$ have the general solutions respectively

$$
\left\{\begin{array}{l}
f(x)=c_{1} \mathrm{e}^{\rho x}+c_{2} \mathrm{e}^{-\rho x}+c_{3} \mathrm{e}^{\mathrm{i} \rho x}+c_{4} \mathrm{e}^{-\mathrm{i} \rho x}+F_{0}(x, \rho), \\
h(x)=d_{1} \mathrm{e}^{\sqrt{\mathrm{i}} \rho x}+d_{2} \mathrm{e}^{-\sqrt{\mathrm{i}} \rho x}+H_{0}(x, \rho),
\end{array}\right.
$$

where $F_{0}(x, \rho)$ and $H_{0}(x, \rho)$ are given by (4.2). Hence, by the boundary conditions of $(4.4), c_{j}, j=1,2,3,4$ and $d_{1}, d_{2}$ satisfy the following algebraic equation:

$$
\left\{\begin{array}{l}
c_{1}+c_{2}+c_{3}+c_{4}=-F_{1}, \\
c_{1} \mathrm{e}^{\rho}+c_{2} \mathrm{e}^{-\rho}+c_{3} \mathrm{e}^{\mathrm{i} \rho}+c_{4} \mathrm{e}^{-\mathrm{i} \rho}=-F_{2}, \\
c_{1} \rho^{2} \mathrm{e}^{\rho}+c_{2} \rho^{2} \mathrm{e}^{-\rho}-c_{3} \rho^{2} \mathrm{e}^{\mathrm{i} \rho}-c_{4} \rho^{2} \mathrm{e}^{-\mathrm{i} \rho}=-F_{3}, \\
d_{1} \mathrm{e}^{\sqrt{\mathrm{i}} \rho}+d_{2} \mathrm{e}^{-\sqrt{\mathrm{i}} \rho}=-H_{4}, \\
c_{1} \rho^{2}+c_{2} \rho^{2}-c_{3} \rho^{2}-c_{4} \rho^{2}-d_{1}-d_{2}=-F_{5}+H_{5}, \\
c_{1} i \rho^{3}-c_{2} i \rho^{3}-c_{3} \rho^{3}+c_{4} \rho^{3}+d_{1} \sqrt{i} \rho-d_{2} \sqrt{i} \rho=-F_{6}-H_{6}+\phi^{\prime}(0) .
\end{array}\right.
$$


Since $\lambda=i \rho^{2} \in \rho(\mathcal{A})$, det $\Delta(\rho) \neq 0$ and hence (4.6) have a unique solution. Moreover, the solution $f(x, \rho)$ and of $h(x, \rho)$ of $(4.5)$ can be written as $(4.3)$.

Proposition 4.2. Let $\mathcal{A}$ be defined by (2.1). Then all $\lambda=i \rho^{2} \in \sigma(\mathcal{A})$ with sufficiently large moduli are algebraically simple.

Proof. We only prove the case when $\rho \in \mathcal{S}$ since the proof for $\bar{\lambda}=-i \bar{\rho}^{2}$ is similar. From Lemma 4.1, the order of each $\lambda \in \sigma(\mathcal{A})$, as a pole of $R(\lambda, \mathcal{A})$, with sufficiently large modulus is less than or equal to the multiplicity of $\lambda$ as a zero of the entire function $\operatorname{det}(\Delta(\rho))$ with respect to $\rho$. Since it is easy to see that $\lambda$ is geometrically simple and from (3.20) and (3.23) all zeros of $\operatorname{det}(\Delta(\rho))=0$ with large moduli are simple in $\mathcal{S}_{1}$ and $\mathcal{S}_{2}$, respectively, the result then follows from the formula: $m_{a} \leq p \cdot m_{g}$ (see e.g. [12], p. 148), where $p$ denotes the order of the pole of the resolvent operator and $m_{a}, m_{g}$ denote the algebraic and geometric multiplicities respectively.

To estimate the norm of the resolvent operator, we recall the Lemma 1.2 of [16] (see also [7]).

Lemma 4.3. Let

$$
D(\lambda)=1+\sum_{i=1}^{n} Q_{i}(\lambda) \mathrm{e}^{\alpha_{i} \lambda}
$$

where $Q_{i}$ are polynomials of $\lambda, \alpha_{i}$ are some complex numbers, and $n$ is a positive integer. Then for all $\lambda$ outside those circles of radius $\varepsilon>0$ that centered at the roots of $D(\cdot)$, one has

$$
|D(\lambda)| \geq C(\varepsilon)>0
$$

for some constant $C(\varepsilon)$ that depends only on $\varepsilon$.

Theorem 4.4. Let $\mathcal{A}$ be defined by (2.1) and for $\lambda \in \rho(\mathcal{A})$, let $R(\lambda, \mathcal{A})=(\lambda-\mathcal{A})^{-1}$ be the resolvent operator of $\mathcal{A}$. Then there exists a constant $M>0$ independent of $\lambda$ such that

$$
\|R(\lambda, \mathcal{A})\| \leq M(1+|\lambda|),
$$

for all $\lambda=i \rho^{2}$ with $\rho \in \mathbb{C}$ lying outside all circles of radius $\epsilon>0$ that are centered at the zeros of $\operatorname{det}(\Delta(\rho))$.

Proof. We first consider those $\lambda=i \rho^{2}$ with $\rho \in \mathcal{S}$. Let $\rho \in \mathcal{S}$ with $\rho \neq 0$. For $(\phi, \psi, \chi) \in \mathcal{H},(f, g, h)=$ $R(\lambda, \mathcal{A})(\phi, \psi, \chi)$ has the expression given by (4.3). In order to estimate $R(\lambda, \mathcal{A})$, since in sector $\mathcal{S}$, we have from (3.3), (3.4) and (3.6) that

$$
\operatorname{Re}(-\rho) \leq \operatorname{Re}(i \rho) \leq 0 \quad \text { and } \quad \operatorname{Re}(-\sqrt{\mathrm{i}} \rho) \leq 0,
$$

we need to use the transformation of the determinant to make the elements $F_{j}, j=1,2, \ldots, 6$ given by (4.1) and (4.1) stable. So, for $F(x, \rho)$ and $H(x, \rho)$ given by (4.1) and (4.1), multiply

$$
\left\{\begin{array}{l}
\text { the first column by }-\frac{1}{8} \rho^{-3} \int_{0}^{1} \mathrm{e}^{-\rho \xi}\left[i \rho^{2} \phi(\xi)+\psi(\xi)\right] \mathrm{d} \xi \\
\text { the second column by }-\frac{1}{8} \rho^{-3} \int_{0}^{1} \mathrm{e}^{\rho \xi}\left[i \rho^{2} \phi(\xi)+\psi(\xi)\right] \mathrm{d} \xi, \\
\text { the third column by } \frac{1}{8} \rho^{-3} \int_{0}^{1} i \mathrm{e}^{-\mathrm{i} \rho \xi}\left[i \rho^{2} \phi(\xi)+\psi(\xi)\right] \mathrm{d} \xi, \\
\text { the fourth column by } \frac{1}{8} \rho^{-3} \int_{0}^{1} i \mathrm{e}^{\mathrm{i} \rho \xi}\left[i \rho^{2} \phi(\xi)+\psi(\xi)\right] \mathrm{d} \xi, \\
\text { the fifth column by }-\frac{1}{4} \rho^{-1} \int_{0}^{1} i \sqrt{i} \mathrm{e}^{-\sqrt{\mathrm{i}} \rho \xi} \chi(\xi) \mathrm{d} \xi, \\
\text { the sixth column by }-\frac{1}{4} \rho^{-1} \int_{0}^{1} i \sqrt{i} \mathrm{e}^{\sqrt{\mathrm{i}} \rho \xi} \chi(\xi) \mathrm{d} \xi,
\end{array}\right.
$$


and add these columns to the last column of $F(x, \rho)$ and $H(x, \rho)$ respectively, we have

$$
F(x, \rho)=\rho^{2} \mathrm{e}^{\rho(1-i+\sqrt{i})} \widetilde{F}(x, \rho), \quad H(x, \rho)=\rho^{4} \mathrm{e}^{\rho(1-i+\sqrt{i})} \widetilde{H}(x, \rho)
$$

where

$$
\widetilde{H}(x, \rho):=\left|\begin{array}{ccccccc}
0 & 0 & 0 & 0 & \mathrm{e}^{\sqrt{\mathrm{i}} \rho x} & \mathrm{e}^{-\sqrt{\mathrm{i}} \rho x} & \widetilde{H_{0}}(x, \rho) \\
\mathrm{e}^{-\rho} & 1 & 1 & \mathrm{e}^{\mathrm{i} \rho} & 0 & 0 & \rho^{-2} \widetilde{F_{1}} \\
1 & \mathrm{e}^{-\rho} & \mathrm{e}^{\mathrm{i} \rho} & 1 & 0 & 0 & \rho^{-2} \widetilde{F}_{2} \\
1 & \mathrm{e}^{-\rho} & -\mathrm{e}^{\mathrm{i} \rho} & -1 & 0 & 0 & \rho^{-2} \widetilde{F}_{3} \\
0 & 0 & 0 & 0 & 1 & \mathrm{e}^{-\sqrt{\mathrm{i}} \rho} & \widetilde{H_{4}} \\
\mathrm{e}^{-\rho} & 1 & -1 & -\mathrm{e}^{\mathrm{i} \rho} & -\rho^{-2} \mathrm{e}^{-\sqrt{\mathrm{i}} \rho} & -\rho^{-2} & \rho^{-2}\left[\widetilde{F_{5}}-\widetilde{H_{5}}\right. \\
i \rho^{2} \mathrm{e}^{-\rho} & -i \rho^{2} & -\rho^{2} & \rho^{2} \mathrm{e}^{\mathrm{i} \rho} & \sqrt{i} \mathrm{e}^{-\sqrt{\mathrm{i}} \rho} & -\sqrt{\mathrm{i}} & \widetilde{F_{6}}+\widetilde{H_{6}}-\phi^{\prime}(0)
\end{array}\right|
$$

and for $s=0,1,2$,

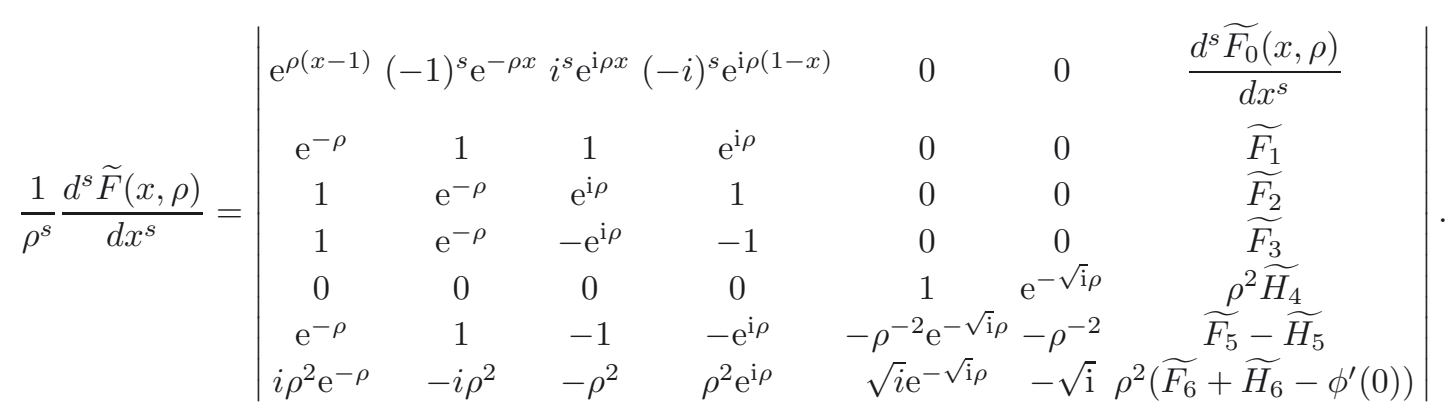

Here

$$
\begin{gathered}
\widetilde{H_{0}}(x, \rho)=\frac{1}{2} \frac{1}{\sqrt{i}}\left[\int_{0}^{x} \mathrm{e}^{-\sqrt{\mathrm{i}} \rho(x-\xi)} \chi(\xi) \mathrm{d} \xi+\int_{x}^{1} \mathrm{e}^{-\sqrt{\mathrm{i}} \rho(\xi-x)} \chi(\xi) \mathrm{d} \xi\right], \\
\frac{d^{s} \widetilde{F_{0}}(x, \rho)}{\mathrm{d} x^{s}}=\frac{1}{4} \int_{0}^{1} \frac{\partial^{s} P(x, \xi)}{\partial x^{s}}\left[i \rho^{2} \phi(\xi)+\psi(\xi)\right] \mathrm{d} \xi, s=0,1,2
\end{gathered}
$$

with

$$
\left\{\begin{array}{c}
P(x, \xi)=\left\{\begin{array}{l}
-\mathrm{e}^{-\rho(x-\xi)}+i \mathrm{e}^{\mathrm{i} \rho(x-\xi)}, x \geq \xi, \\
-\mathrm{e}^{-\rho(\xi-x)}+i \mathrm{e}^{\mathrm{i} \rho(\xi-x)}, x<\xi,
\end{array}\right. \\
\frac{\partial P(x, \xi)}{\partial x}= \begin{cases}\mathrm{e}^{-\rho(x-\xi)}-\mathrm{e}^{\mathrm{i} \rho(x-\xi)}, & x \geq \xi, \\
-\mathrm{e}^{-\rho(\xi-x)}+\mathrm{e}^{\mathrm{i} \rho(\xi-x)}, & x<\xi,\end{cases} \\
\frac{\partial^{2} P(x, \xi)}{\partial x^{2}}= \begin{cases}-\mathrm{e}^{-\rho(x-\xi)}-i \mathrm{e}^{\mathrm{i} \rho(x-\xi)}, & x \geq \xi, \\
-\mathrm{e}^{-\rho(\xi-x)}-i \mathrm{e}^{\mathrm{i} \rho(\xi-x)}, & x<\xi,\end{cases}
\end{array}\right.
$$


and

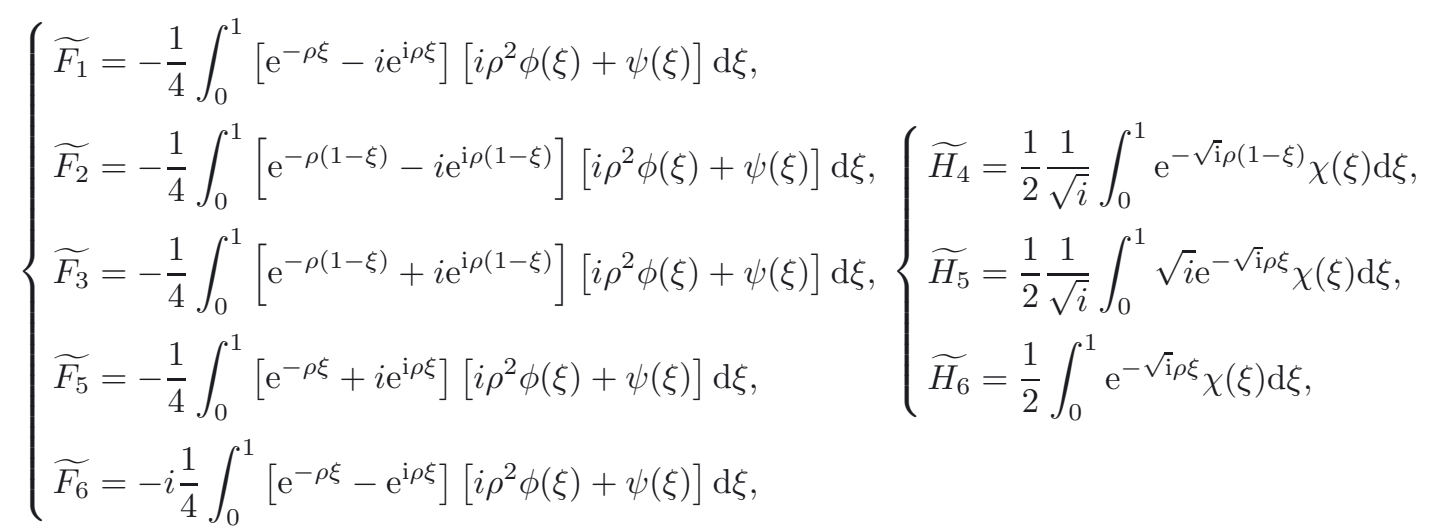

So, by (3.2)-(3.6), the asymptotic expression of $\operatorname{det} \Delta(\rho)$ given by (3.10) in $\mathcal{S},(3.12)$ in $\mathcal{S}_{1}$, and (3.13) in $\mathcal{S}_{2}$, respectively, and Lemma 4.3 , there is $M_{1}>0$, such that

$$
\begin{gathered}
\left|f^{\prime \prime}(x)\right| \leq \frac{M_{1}}{|\rho|}\left[\int_{0}^{1}[|\lambda||\phi(\xi)|+|\psi(\xi)|+|\chi(\xi)|] \mathrm{d} \xi+\left|\phi^{\prime}(0)\right|\right] \\
|g(x)| \leq \frac{M_{1}}{|\rho|}\left[\int_{0}^{1}[|\lambda||\phi(\xi)|+|\psi(\xi)|+|\chi(\xi)|] \mathrm{d} \xi+\left|\phi^{\prime}(0)\right|\right]+|\phi(x)|, \\
|h(x)| \leq \frac{M_{1}}{|\rho|} \int_{0}^{1}[|\lambda||\phi(\xi)|+|\psi(\xi)|+|\chi(\xi)|] \mathrm{d} \xi
\end{gathered}
$$

for all $\lambda=i \rho^{2}$ with $\rho \in \mathcal{S}$ lying outside all circles of radius $\epsilon>0$ that are centered at the zeros of $\operatorname{det}(\Delta(\rho))$. Since $\left|l^{\prime}(x)\right| \leq\left\|l^{\prime \prime}\right\|_{L^{2}}$ and $|l(x)| \leq\left\|l^{\prime}\right\|_{L^{2}} \leq\left\|l^{\prime \prime}\right\|_{L^{2}}$ for any $x \in[0,1]$ and $l \in H_{L}^{2}[0,1]$, it follows that $\forall(\phi, \psi, \chi) \in \mathcal{H}$,

$$
\left\{\begin{array}{l}
|\lambda|^{-1}\left|f^{\prime \prime}(x)\right| \leq \frac{M_{1}}{|\rho|}\left[\left\|\phi^{\prime \prime}\right\|_{L^{2}}+|\lambda|^{-1}\|\psi\|_{L^{2}}+|\lambda|^{-1}\|\chi\|_{L^{2}}+|\lambda|^{-1}\left\|\phi^{\prime \prime}\right\|_{L^{2}}\right] \\
|\lambda|^{-1}|g(x)| \leq \frac{M_{1}}{|\rho|}\left[\left\|\phi^{\prime \prime}\right\|_{L^{2}}+|\lambda|^{-1}\|\psi\|_{L^{2}}+|\lambda|^{-1}\|\chi\|_{L^{2}}+|\lambda|^{-1}\left\|\phi^{\prime \prime}\right\|_{L^{2}}\right]+|\lambda|^{-1}\left\|\phi^{\prime \prime}\right\|_{L^{2}} \\
|\lambda|^{-1}|h(x)| \leq \frac{M_{1}}{|\rho|}\left[\left\|\phi^{\prime \prime}\right\|_{L^{2}}+|\lambda|^{-1}\|\psi\|_{L^{2}}+|\lambda|^{-1}\|\chi\|_{L^{2}}\right]
\end{array}\right.
$$

It is seen from the above that we can find constants $M_{2}, K>0$ independent of $\lambda$ such that

$$
\|(f, g, h)\| \leq M_{2}(1+|\lambda|)\|(\phi, \psi, \chi)\|
$$

for all $|\lambda|=\left|\rho^{2}\right|>K>1$ with $\rho \in \mathcal{S}$ lies outside all circles of radius $\epsilon>0$ that centered at the zeros of $\operatorname{det}(\Delta(\rho))$. Moreover, there is $M>M_{2}$ such that for $|\lambda| \leq K$, we have $\|(f, g, h)\| \leq M\|(\phi, \psi, \chi)\|$. Therefore, we get

$$
\|(f, g, h)\| \leq M(1+|\lambda|)\|(\phi, \psi, \chi)\|
$$

for all $\lambda=i \rho^{2}$ with $\rho \in \mathcal{S}$ lying outside all circles of radius $\epsilon>0$ that are centered at the zeros of $\operatorname{det}(\Delta(\rho))$.

This result can be extended to all the other $\rho$ 's by the exact same arguments of ([13], pp. 56-60).

Theorem 4.5. Let $\mathcal{A}$ be defined by (2.1). Then both the root subspaces of $\mathcal{A}$ and $\mathcal{A}^{*}$ are complete in $\mathcal{H}$, that is, $\operatorname{Sp}\left(\mathcal{A}^{*}\right)=\operatorname{Sp}(\mathcal{A})=\mathcal{H}$. 
Proof. We only show the completeness for the root subspace of $\mathcal{A}$ since the proof for that of $\mathcal{A}^{*}$ is almost the same. It follows from Lemma 5 on page 2355 of [2] that the following orthogonal decomposition holds:

$$
\mathcal{H}=\sigma_{\infty}\left(\mathcal{A}^{*}\right) \oplus \operatorname{Sp}(\mathcal{A})
$$

where $\sigma_{\infty}\left(\mathcal{A}^{*}\right)$ consists of those $Y \in \mathcal{H}$ so that $R\left(\lambda, \mathcal{A}^{*}\right) Y$ is an analytic function of $\lambda$ in the whole complex plane. Hence, $\operatorname{Sp}(\mathcal{A})=\mathcal{H}$ if and only if $\sigma_{\infty}\left(\mathcal{A}^{*}\right)=\{0\}$. Now suppose that $Y \in \sigma_{\infty}\left(\mathcal{A}^{*}\right)$. Since $R\left(\lambda, \mathcal{A}^{*}\right) Y$ is an analytic function in $\lambda$, it is also analytic in $\rho$. By the maximum modulus principle (or the Phragmén-Lindelöf's theorem) and the fact that $\left\|R\left(\lambda, \mathcal{A}^{*}\right)\right\|=\|R(\bar{\lambda}, \mathcal{A})\|$, it follows from Theorem 4.4 that

$$
\left\|R\left(\lambda, \mathcal{A}^{*}\right) Y\right\| \leq M(1+|\lambda|)\|Y\|, \forall \lambda \in \mathbb{C},
$$

for some constant $M>0$. By Theorem 1 of ([9], p. 3), we conclude that $R\left(\lambda, \mathcal{A}^{*}\right) Y$ is a polynomial in $\lambda$ of degree $\leq 1$, i.e., $R\left(\lambda, \mathcal{A}^{*}\right) Y=Y_{0}+\lambda Y_{1}$ for some $Y_{0}, Y_{1} \in \mathcal{H}$. Thus $Y=\left(\lambda-\mathcal{A}^{*}\right)\left(Y_{0}+\lambda Y_{1}\right)$. Since $\mathcal{A}^{*}$ is a closed operator, $Y_{1}$ belongs to $D\left(\mathcal{A}^{*}\right)$ and so does $Y_{0}$. Therefore,

$$
-\mathcal{A}^{*} Y_{0}+\lambda\left(Y_{0}-\mathcal{A}^{*} Y_{1}\right)+\lambda^{2} Y_{1}=Y, \forall \lambda \in \mathbb{C} .
$$

Comparing the coefficients of $\lambda^{2}, \lambda$ and $\lambda^{0}$ in two sides of the above equation, we get $Y_{1}=Y_{0}=Y=0$.

\section{RIESZ BASIS PROPERTY AND EXPONENTIAL STABILITY}

In this section, we show the Riesz basis generation and exponential stability of the system (2.2). To establish the Riesz basis property of the system (2.2), we recall the following two lemmas:

Lemma 5.1. An approximately normalized sequence $\left\{e_{i}\right\}_{i=1}^{\infty}$ and its approximately normalized biorthogonal sequence $\left\{e_{i}^{*}\right\}_{i=1}^{\infty}$ are Riesz bases for a Hilbert space $H$ if and only if ([22], p. 27)

(a) both $\left\{e_{i}\right\}_{i=1}^{\infty}$ and $\left\{e_{i}^{*}\right\}_{i=1}^{\infty}$ are complete in $H$; and

(b) both $\left\{e_{i}\right\}_{i=1}^{\infty}$ and $\left\{e_{i}^{*}\right\}_{i=1}^{\infty}$ are Bessel sequences in $H$, that is, for any $f \in H$, two sequences $\left\{\left\langle f, e_{i}\right\rangle\right\}_{i=1}^{\infty}$, $\left\{\left\langle f, e_{i}^{*}\right\rangle\right\}_{i=1}^{\infty}$ belong to $\ell^{2}$.

Lemma 5.2 ([16], Lem. 3.2). Let $\left\{\mu_{n}\right\}$ be a sequence which has asymptotics

$$
\mu_{n}=\alpha(n+i \beta \ln n)+\mathcal{O}(1), \quad \alpha \neq 0, n=1,2,3, \ldots,
$$

where $\beta$ is a real number. If $\mu_{n}$ satisfies $\sup _{n>1} \operatorname{Re} \mu_{n}<\infty$, then the sequence $\left\{\mathrm{e}^{\mu_{n} x}\right\}_{n=1}^{\infty}$ is a Bessel sequence in $L^{2}(0,1)$.

Lemma 5.3. Let $\varphi_{n j}^{p}(x)$ and $\varphi_{n j}^{e}(x), j=1,2,3,4$, be given by (3.27) and (3.30), respectively. Then all $\left\{\varphi_{n j}^{p}(x)\right\}_{n=1}^{\infty}$ and $\left\{\varphi_{n j}^{e}(x)\right\}_{n=1}^{\infty}, j=1,2,3,4$, are Bessel sequences in $L^{2}(0,1)$.

Proof. By (3.27), if we take $\alpha=i \sqrt{i} \pi, \beta=0$ in $\varphi_{n 1}^{p}(x), \alpha=-\sqrt{\mathrm{i}} \pi, \beta=0$ in $\varphi_{n 2}^{p}(x), \alpha=i \pi, \beta=0$ in $\varphi_{n 3}^{p}(x)$, and $\alpha=-i \pi, \beta=0$ in $\varphi_{n 4}^{p}(x)$, respectively, then it follows from Lemma 5.2 directly that $\left\{\varphi_{n j}^{p}(x)\right\}_{n=1}^{\infty}, j=1,2,3,4$, are four Bessel sequences in $L^{2}(0,1)$.

Similarly, by (3.30), if we take $\alpha=i \pi, \beta=0$ in $\varphi_{n 1}^{e}(x), \alpha=-i \pi, \beta=0$ in $\varphi_{n 2}^{e}(x), \alpha=-\pi, \beta=0$ in $\varphi_{n 3}^{e}(x)$, and $\alpha=-\sqrt{\mathrm{i}} \pi, \beta=0$ in $\varphi_{n 4}^{e}(x)$, respectively, then it follows from Lemma 5.2 directly that $\varphi_{n j}^{e}(x), j=1,2,3,4$, are Bessel sequences in $L^{2}(0,1)$.

Now we can establish the Riesz basis property of the system (2.2). 
Theorem 5.4. Let $\mathcal{A}$ be defined by (2.1). Then the generalized eigenfunctions of $\mathcal{A}$ form a Riesz basis for $\mathcal{H}$.

Proof. Let $\sigma(\mathcal{A})=\left\{\lambda_{n}^{p}, \lambda_{n}^{e}, \overline{\lambda_{2 n}^{e}}\right\}_{n=1}^{\infty}$ be the eigenvalues of $\mathcal{A}$. By Theorem 3.3 and Proposition 4.2, we have that each eigenvalue of $\mathcal{A}$ with sufficient large modulus is simple, and hence there exists an integer $N>0$ such that all $\lambda_{n}^{p}, \lambda_{n}^{e}, \overline{\lambda_{2 n}^{e}}$ with $n \geq N$, are algebraically simple. For $n \leq N$, if the algebraic multiplicities of $\lambda_{n}^{p}$ and $\lambda_{n}^{e}$ are $m_{n}^{p}$ and $m_{n}^{e}$, respectively, we can find the highest order generalized eigenfunctions $\Phi_{n, 1}^{p}$ and $\Phi_{n, 1}^{e}$ from respectively

$$
\left(\mathcal{A}-\lambda_{n}^{p}\right)^{m_{n}^{p}} \Phi_{n, 1}^{p}=0, \quad\left(\mathcal{A}-\lambda_{n}^{p}\right)^{m_{n}^{p}-1} \Phi_{n, 1}^{p} \neq 0
$$

and

$$
\left(\mathcal{A}-\lambda_{n}^{e}\right)^{m_{n}^{e}} \Phi_{n, 1}^{e}=0, \quad\left(\mathcal{A}-\lambda_{n}^{e}\right)^{m_{n}^{e}-1} \Phi_{n, 1}^{e} \neq 0 .
$$

Furthermore, the other lower order linearly independent generalized eigenfunctions associated with $\lambda_{n}^{p}$ and $\lambda_{n}^{e}$ can be found through $\Phi_{n, j}^{p}=\left(\mathcal{A}-\lambda_{n}^{p}\right)^{j-1} \Phi_{n, 1}^{p}, j=2,3, \ldots, m_{n}^{p}$, and $\Phi_{n, s}^{e}=\left(\mathcal{A}-\lambda_{n}^{e}\right)^{s-1} \Phi_{n, 1}^{e}, s=2,3, \ldots, m_{n}^{e}$, respectively, where $\Phi_{n, m_{n}^{p}}^{p}$ and $\Phi_{n, m_{n}^{e}}^{e}$ are eigenfunctions of $\mathcal{A}$ with respect to $\lambda_{n}^{p}$ and $\lambda_{n}^{e}$, respectively. Assume $\Phi_{n}^{p}$ and $\Phi_{n}^{e}$ are the normalized eigenfunctions of $\mathcal{A}$ corresponding to $\lambda_{n}^{p}$ and $\lambda_{n}^{e}$ with $n \geq N$ respectively. Then

$$
\left\{\left\{\Phi_{n, j}^{p}\right\}_{j=1}^{m_{n}^{p}}\right\}_{n<N} \cup\left\{\Phi_{n}^{p}\right\}_{n \geq N} \bigcup\left\{\left\{\Phi_{n, j}^{e}, \overline{\Phi_{n, j}^{e}}\right\}_{j=1}^{m_{n}^{e}}\right\}_{n<N} \cup\left\{\Phi_{n}^{e}, \overline{\Phi_{n}^{e}}\right\}_{n \geq N}
$$

are all linearly independent generalized eigenfunctions of $\mathcal{A}$. On the other hand, we also have that

$$
\left\{\left\{\Psi_{n, j}^{p}\right\}_{j=1}^{m_{n}^{p}}\right\}_{n<N} \cup\left\{\Psi_{n}^{p}\right\}_{n \geq N} \bigcup\left\{\left\{\Psi_{n, j}^{e}, \overline{\Psi_{n, j}^{e}}\right\}_{j=1}^{m_{n}^{e}}\right\}_{n<N} \cup\left\{\Psi_{n}^{e}, \overline{\Psi_{n}^{e}}\right\}_{n \geq N}
$$

are all linearly independent generalized eigenfunctions of $\mathcal{A}^{*}$. Let

$$
\left\{\begin{array}{l}
\Phi_{n, j}^{p *}=\frac{\Psi_{n, j}^{p}}{\overline{\left\langle\Phi_{n, j}^{p}, \Psi_{n, j}^{p}\right\rangle}}, n<N, j=1,2, \ldots, m_{n}^{p}, \\
\Phi_{n}^{p *}=\frac{\Psi_{n}^{p}}{\left\langle\Phi_{n}^{p}, \Psi_{n}^{p}\right\rangle}, \quad n \geq N
\end{array}\right.
$$

and

Then

$$
\left\{\begin{array}{l}
\Phi_{n, j}^{e *}=\frac{\overline{\Psi_{n, j}^{e}}}{\left.\overline{\left\langle\Phi_{n, j}^{e}, \overline{\Psi_{n, j}^{e}}\right.}\right\rangle}, n<N, j=1,2, \ldots, m_{n}^{e}, \\
\Phi_{n}^{e *}=\frac{\overline{\Psi_{n}^{e}}}{\overline{\left\langle\Phi_{n}^{e}, \overline{\Psi_{n}^{e}}\right\rangle}}, \quad n \geq N .
\end{array}\right.
$$

$$
\left\{\left\{\Phi_{n, j}^{p *}\right\}_{j=1}^{m_{n}^{p}}\right\}_{n<N} \cup\left\{\Phi_{n}^{p *}\right\}_{n \geq N} \bigcup\left\{\left\{\Phi_{n, j}^{e *}, \overline{\Phi_{n, j}^{e *}}\right\}_{j=1}^{m_{n}^{e}}\right\}_{n<N} \cup\left\{\Phi_{n}^{e *}, \overline{\Phi_{n}^{e *}}\right\}_{n \geq N}
$$

are all linearly independent generalized eigenfunctions of $\mathcal{A}^{*}$ and they are bi-orthogonal to the sequence given by (5.2). Actually, it is easy to see that

$$
\begin{cases}\left\langle\Phi_{s, i}^{p}, \Phi_{l, j}^{p *}\right\rangle=\delta_{s l} \times \delta_{i j}, & \text { for } 1 \leq s, l<N, 1 \leq i \leq m_{s}^{p}, 1 \leq j \leq m_{l}^{p}, \\ \left\langle\Phi_{s, i}^{e}, \Phi_{l, j}^{e *}\right\rangle=\delta_{s l} \times \delta_{i j}, & \text { for } 1 \leq s, l<N, 1 \leq i \leq m_{s}^{e}, 1 \leq j \leq m_{l}^{e}, \\ \left\langle\Phi_{s}^{p}, \Phi_{l}^{p *}\right\rangle=\delta_{s l},\left\langle\Phi_{s}^{e}, \Phi_{l}^{e *}\right\rangle=\delta_{s l}, & \text { for } N \leq s, l<\infty, \\ \left\langle\Phi_{s, i}^{p}, \Phi_{l, j}^{e *}\right\rangle=\left\langle\Phi_{s, i}^{p}, \overline{\Phi_{l, j}^{e *}}\right\rangle=\left\langle\Phi_{s, i}^{e}, \Phi_{l, j}^{p *}\right\rangle=\left\langle\overline{\Phi_{s, i}^{e}}, \Phi_{l, j}^{p *}\right\rangle=0, & \text { for } 1 \leq s, l<N, 1 \leq i \leq m_{s}^{p}, 1 \leq j \leq m_{l}^{p}, \\ \left\langle\Phi_{s}^{p}, \Phi_{l}^{e *}\right\rangle=\left\langle\Phi_{s}^{p}, \overline{\Phi_{l}^{e *}}\right\rangle=\left\langle\Phi_{s}^{e}, \Phi_{l}^{p *}\right\rangle=\left\langle\overline{\Phi_{s}^{e}}, \Phi_{l}^{p *}\right\rangle=0, & \text { for } N \leq s, l<\infty, \\ \left\langle\Phi_{s, i}^{p}, \Phi_{l}^{e *}\right\rangle=\left\langle\Phi_{s, i}^{p}, \overline{\Phi_{l}^{e *}}\right\rangle=0, & \text { for } 1 \leq s<N, N \leq l<\infty, 1 \leq i \leq m_{s}^{p}, \\ \left\langle\Phi_{s, j}^{e}, \Phi_{l}^{p *}\right\rangle=\left\langle\overline{\Phi_{s, j}^{e}}, \Phi_{l}^{p *}\right\rangle=0, & \text { for } 1 \leq s<N, N \leq l<\infty, 1 \leq j \leq m_{e}^{p},\end{cases}
$$


where $\delta_{i j}$ is Kronecker delta and satisfies

$$
\delta_{i j}=\left\{\begin{array}{l}
1, i=j \\
0, i \neq j
\end{array}\right.
$$

Hence, the set (5.6) is the bi-orthogonal sequence of (5.2). From Theorem 4.5, we have that all the sequences given by (5.2), (5.3) and (5.6) are complete in $\mathcal{H}$.

Hence, in order to prove the Riesz basis property of the system, since both $\left\{\left\{\Phi_{n, j}^{p}\right\}_{j=1}^{m_{n}^{p}},\left\{\Phi_{n, j}^{e}\right\}_{j=1}^{m_{n}^{e}}\right\}_{n<N}$ and $\left\{\left\{\Phi_{n, j}^{p *}\right\}_{j=1}^{m_{n}^{p}},\left\{\Phi_{n, j}^{e *}\right\}_{j=1}^{m_{n}^{e}}\right\}_{n<N}$ are finitely many, it suffices to show that both the eigenfunctions $\left\{\Phi_{n}^{p}, \Phi_{n}^{e}\right\}_{n \geq N}$ and $\left\{\Phi_{n}^{p *}, \Phi_{n}^{e *}\right\}_{n \geq N}$ of $\mathcal{A}$ and $\mathcal{A}^{*}$, respectively, are Bessel sequences in $\mathcal{H}$. Moreover, it follows from (5.4) and (5.5), that $\left\{\Phi_{n}^{p *}, \Phi_{n}^{e *}\right\}_{n \geq N}$ is a Bessel sequence if and only if $\left\{\Psi_{n}^{p}, \Psi_{n}^{e}\right\}_{n \geq N}$ is a Bessel sequence. So we only need to show that $\left\{\Phi_{n}^{p}, \Phi_{n}^{e}\right\}_{n \geq N}$ and $\left\{\Psi_{n}^{p}, \Psi_{n}^{e}\right\}_{n \geq N}$ are Bessel sequences in $\mathcal{H}$.

Without loss of generality, we may assume that $\Phi_{n}^{p}=\left(f_{n}^{p}, \lambda_{n}^{p} f_{n}^{p}, h_{n}^{p}\right), \Phi_{n}^{e}=\left(f_{n}^{e}, \lambda_{n}^{e} f_{n}^{e}, h_{n}^{e}\right)$ and $\Psi_{n}^{p}=$ $\left(f_{n}^{p},-\lambda_{n}^{p} f_{n}^{p}, h_{n}^{p}\right), \Psi_{n}^{e}=\left(f_{n}^{e},-\lambda_{n}^{e} f_{n}^{e}, h_{n}^{e}\right)$ given by (3.26), (3.29) and (3.35), (3.36), respectively, for all $n \geq N$. It then follows from Lemma 5.3 and the expansions of (3.26), (3.29) and (3.35), (3.36) that all of $\left\{\left(f_{n}^{p}\right)^{\prime \prime}\right\}_{n=N}^{\infty}$, $\left\{\left(f_{n}^{e}\right)^{\prime \prime}\right\}_{n=N}^{\infty},\left\{ \pm \lambda_{n}^{p} f_{n}^{p}\right\}_{n=N}^{\infty},\left\{ \pm \lambda_{n}^{e} f_{n}^{e}\right\}_{n=N}^{\infty},\left\{h_{n}^{p}\right\}_{n=N}^{\infty}$ and $\left\{h_{n}^{e}\right\}_{n=N}^{\infty}$ are Bessel sequences in $L^{2}(0,1)$. Hence both of $\left\{\Phi_{n}^{p}, \Phi_{n}^{e}\right\}_{n \geq N}$ and $\left\{\Psi_{n}^{p}, \Psi_{n}^{e}\right\}_{n \geq N}$ are also Bessel sequences in $\mathcal{H}$. Therefore we get that both of $\left\{\Phi_{n}^{p}, \Phi_{n}^{e}\right\}_{n \geq N}$ and $\left\{\Phi_{n}^{p *}, \Phi_{n}^{e *}\right\}_{n \geq N}$ are also Bessel sequences in $\mathcal{H}$. The desired result of the theorem then follows from Lemma 5.1.

Theorem 5.5. Let $\mathcal{A}$ be defined by (2.1). Then the spectrum-determined growth condition $\omega(\mathcal{A})=s(\mathcal{A})$ holds true, where $\omega(\mathcal{A})$ is the growth bound of the $C_{0}$-semigroup $\mathrm{e}^{\mathcal{A} t}$ and

$$
s(\mathcal{A}):=\sup \{\operatorname{Re} \lambda \mid \lambda \in \sigma(\mathcal{A})\}
$$

is the spectral bound of $\mathcal{A}$. Moreover, the system (2.2) is exponentially stable, that is, there exist two positive constants $M$ and $\omega$ such that the $C_{0}$-semigroup $\mathrm{e}^{\mathcal{A} t}$ generated by $\mathcal{A}$ satisfies

$$
\left\|\mathrm{e}^{\mathcal{A} t}\right\| \leq M \mathrm{e}^{-\omega t} .
$$

Proof. The spectrum-determined growth condition follows from Theorem 5.4. By Lemma 3.1, for each $\lambda \in \sigma(\mathcal{A})$, we have $\operatorname{Re} \lambda<0$. This, together with (3.16)-(3.19) and the spectrum-determined growth condition, shows that $\mathrm{e}^{\mathcal{A} t}$ is exponentially stable.

\section{Gevrey REgularity}

In what follows, we show that the $C_{0}$-semigroup $\mathrm{e}^{\mathcal{A} t}$ generated by $\mathcal{A}$ is of a Gevrey class $\delta$ with any $\delta>2$. We recall the definition.

Definition 6.1 ([1,18]). A $C_{0}$-semigroup $T(t)$ is of a Gevrey class $\delta>1$ for $t>t_{0}$ if $T(t)$ is infinitely differentiable for $t>t_{0}$ and for every compact subset $K \subset\left(t_{0}, \infty\right)$ and each $\theta>0$, there is a constant $C=C(K, \theta)$ such that

$$
\left\|T^{(n)}(t)\right\| \leq C \theta^{n}(n !)^{\delta}, \quad \forall t \in K, n=0,1,2, \ldots
$$

In order to get the Gevrey regularity of the system (2.2), we need the following theorem established by Taylor in ([18], Thm. 4, Chap. 5).

Theorem 6.2. Let $\mathrm{e}^{\mathcal{A} t}$ be a $C_{0}$-semigroup satisfying $\left\|\mathrm{e}^{\mathcal{A} t}\right\| \leq M \mathrm{e}^{\omega t}$. Suppose that for some $\mu \geq \omega$ and $\alpha$ satisfying $0<\alpha \leq 1$,

$$
\lim _{|\tau| \rightarrow \infty} \sup |\tau|^{\alpha}\|R(\mu+i \tau, \mathcal{A})\|=C<\infty, \quad \tau \in \mathbb{R} .
$$

Then $\mathrm{e}^{\mathcal{A} t}$ is of Gevrey class $\delta$ with $\delta>1 / \alpha$ for $t>0$.

Now we establish the Gevrey regularity of the system (2.2). 
Theorem 6.3. Let $\mathcal{A}$ be defined by (2.1). Then the semigroup $\mathrm{e}^{\mathcal{A} t}$, generated by $\mathcal{A}$, is of a Gevrey class $\delta>2$ with $t_{0}=0$.

Proof. From Theorem 5.5, $\mathcal{A}$ generates a exponentially stable $C_{0}$-semigroup e $\mathrm{e}^{\mathcal{A} t}$ in $\mathcal{H}$. So, by Theorem 6.2 , we only need to show

$$
\lim _{|\tau| \rightarrow \infty}|\tau|\|R(i \tau, \mathcal{A})\|^{2}=C<\infty, \quad \tau \in \mathbb{R} .
$$

By Theorem 5.4,

$$
\left\{\left\{\Phi_{n, j}^{p}\right\}_{j=1}^{m_{n}^{p}}\right\}_{n<N} \cup\left\{\Phi_{n}^{p}\right\}_{n \geq N} \bigcup\left\{\left\{\Phi_{n, j}^{e}, \overline{\Phi_{n, j}^{e}}\right\}_{j=1}^{m_{n}^{e}}\right\}_{n<N} \cup\left\{\Phi_{n}^{e}, \overline{\Phi_{n}^{e}}\right\}_{n \geq N}
$$

forms a Riesz basis in $\mathcal{H}$. Then for each $Y \in \mathcal{H}$, we have

$$
Y=\sum_{n=1}^{N-1} \sum_{j=1}^{m_{n}^{p}} a_{n, j}^{p} \Phi_{n, j}^{p}+\sum_{n=N}^{\infty} a_{n}^{p} \Phi_{n}^{p}+\sum_{n=1}^{N-1} \sum_{j=1}^{m_{n}^{e}}\left[a_{n, j}^{e} \Phi_{n, j}^{e}+b_{n, j}^{e} \overline{\Phi_{n, j}^{e}}\right]+\sum_{n=N}^{\infty}\left[a_{n}^{e} \Phi_{n}^{e}+b_{n}^{e} \overline{\Phi_{n}^{e}}\right],
$$

and

$$
\|Y\|^{2} \asymp \sum_{n=1}^{N-1} \sum_{j=1}^{m_{n}^{p}}\left|a_{n, j}^{p}\right|^{2}+\sum_{n=N}^{\infty}\left|a_{n}^{p}\right|^{2}+\sum_{n=1}^{N-1} \sum_{j=1}^{m_{n}^{e}}\left[\left|a_{n, j}^{e}\right|^{2}+\left|b_{n, j}^{e}\right|^{2}\right]+\sum_{n=N}^{\infty}\left[\left|a_{n}^{e}\right|^{2}+\left|b_{n}^{e}\right|^{2}\right] .
$$

Let $\tau>0$. Then we have $i \tau \in \rho(\mathcal{A})$, and, in addition,

$$
\begin{aligned}
R(i \tau, \mathcal{A}) Y= & \sum_{n=1}^{N-1} \sum_{j=1}^{m_{n}^{p}} \frac{a_{n, j}^{p} \Phi_{n, j}^{p}}{i \tau-\lambda_{n}^{p}}+\sum_{n=1}^{N-1} \sum_{j=1}^{m_{n}^{e}}\left[\frac{a_{n, j}^{e} \Phi_{n, j}^{e}}{i \tau-\lambda_{n}^{e}}+\frac{b_{n, j}^{e} \overline{\Phi_{n, j}^{e}}}{i \tau-\overline{\lambda_{n}^{e}}}\right]+\sum_{n=N}^{\infty}\left[\frac{a_{n}^{e} \Phi_{n}^{e}}{i \tau-\lambda_{n}^{e}}+\frac{b_{n}^{e} \overline{\Phi_{n}^{e}}}{i \tau-\overline{\lambda_{n}^{e}}}\right] \\
& +\sum_{n=N}^{\infty} \frac{a_{n}^{p} \Phi_{n}^{p}}{i \tau-\lambda_{n}^{p}}+\sum_{n=1}^{N-1} \mathcal{O}\left(\frac{1}{\left|i \tau-\lambda_{n}^{p}\right|^{2}}\right)+\sum_{n=1}^{N-1}\left[\mathcal{O}\left(\frac{1}{\left|i \tau-\lambda_{n}^{e}\right|^{2}}\right)+\mathcal{O}\left(\frac{1}{\left|i \tau-\overline{\lambda_{n}^{e}}\right|^{2}}\right)\right]
\end{aligned}
$$

and

$$
\begin{aligned}
\|R(i \tau, \mathcal{A}) Y\|^{2} \asymp & \sum_{n=1}^{N-1} \sum_{j=1}^{m_{n}^{p}} \frac{\left|a_{n, j}^{p}\right|^{2}}{\left|i \tau-\lambda_{n}^{p}\right|^{2}}+\sum_{n=N}^{\infty} \frac{\left|a_{n}^{p}\right|^{2}}{\left|i \tau-\lambda_{n}^{p}\right|^{2}} \\
& +\sum_{n=1}^{N-1} \sum_{j=1}^{m_{n}^{e}}\left[\frac{\left|a_{n, j}^{e}\right|^{2}}{\left|i \tau-\lambda_{n}^{e}\right|^{2}}+\frac{\left|b_{n, j}^{e}\right|^{2}}{\left|i \tau-\overline{\lambda_{n}^{e}}\right|^{2}}\right]+\sum_{n=N}^{\infty}\left[\frac{\left|a_{n}^{e}\right|^{2}}{\left|i \tau-\lambda_{n}^{e}\right|^{2}}+\frac{\left|b_{n}^{e}\right|^{2}}{\left|i \tau-\overline{\lambda_{n}^{e}}\right|^{2}}\right],
\end{aligned}
$$

where $\left\{\lambda_{n}^{p}, n \in \mathbb{N}\right\}$ and $\left\{\lambda_{n}^{e}, \overline{\lambda_{n}^{e}}, n \in \mathbb{N}\right\}$, given by (3.17), are eigenvalues of $\mathcal{A}$.

Now we estimate $\left|i \tau-\lambda_{n}^{p}\right|^{2},\left|i \tau-\lambda_{n}^{e}\right|^{2}$ and $\left|i \tau-\overline{\lambda_{n}^{e}}\right|^{2}$. By (3.17), for $n$ large enough, we have

$$
\left|i \tau-\lambda_{n}^{p}\right|^{2}=\left|i \tau+\left[n \pi+\frac{1}{2} \theta_{p}\right]^{2}+\mathcal{O}\left(\mathrm{e}^{-k_{1} n}\right)\right|^{2}=\tau^{2}+\left[n \pi+\frac{1}{2} \theta_{p}\right]^{4}+\mathcal{O}\left(\mathrm{e}^{-\left(k_{1}-\varepsilon\right) n}\right) \geq M_{1} \tau^{2}
$$

where $\varepsilon$ denotes a small positive constant, and

$$
\begin{gathered}
\left|i \tau-\lambda_{n}^{e}\right|^{2}=\left|\tau+i \lambda_{n}^{e}\right|^{2}=\left|\tau-\left(\rho_{n}^{e}\right)^{2}\right|^{2}=\left|\sqrt{\tau}+\rho_{n}^{e}\right|^{2}\left|\sqrt{\tau}-\rho_{n}^{e}\right|^{2}, \\
\left|i \tau-\overline{\lambda_{n}^{e}}\right|^{2}=\left|\tau+i \overline{\lambda_{n}^{e}}\right|^{2}=\left|\tau-i^{2} \overline{\left(\rho_{n}^{e}\right)^{2}}\right|^{2}=\left|\sqrt{\tau}+i \overline{\rho_{n}^{e}}\right|^{2}\left|\sqrt{\tau}-i \overline{\rho_{n}^{e}}\right|^{2},
\end{gathered}
$$


where $\lambda_{n}^{e}=i\left(\rho_{n}^{e}\right)^{2}$ with $\rho_{n}^{e}$ given by (3.25), and $M_{1}>0$ is a constant. Noting that

$$
\begin{aligned}
\left|\sqrt{\tau}+\rho_{n}^{e}\right|^{2} & =\left|\sqrt{\tau}+\left(n \pi+\frac{1}{2} \theta_{e}\right)+\frac{1}{2 i} \ln r+\mathcal{O}\left(\mathrm{e}^{-k_{2} n}\right)\right|^{2} \\
& =\left[\sqrt{\tau}+n \pi+\frac{1}{2} \theta_{e}\right]^{2}+\frac{1}{4}(\ln r)^{2}+\mathcal{O}\left(\mathrm{e}^{-\left(k_{2}-\varepsilon\right) n}\right) \\
\left|\sqrt{\tau}-\rho_{n}^{e}\right|^{2} & =\left|\sqrt{\tau}-\left(n \pi+\frac{1}{2} \theta_{e}\right)-\frac{1}{2 i} \ln r+\mathcal{O}\left(\mathrm{e}^{-k_{2} n}\right)\right|^{2} \\
& =\left[\sqrt{\tau}-\left(n \pi+\frac{1}{2} \theta_{e}\right)\right]^{2}+\frac{1}{4}(\ln r)^{2}+\mathcal{O}\left(\mathrm{e}^{-\left(k_{2}-\varepsilon\right) n}\right) \\
\left|\sqrt{\tau}+i \overline{\rho_{n}^{e}}\right|^{2} & =\left|\sqrt{\tau}-\frac{1}{2} \ln r+\left(n \pi+\frac{1}{2} \theta_{e}\right) i+\mathcal{O}\left(\mathrm{e}^{-k_{2} n}\right)\right|^{2} \\
& =\left[\sqrt{\tau}+\frac{1}{2}|\ln r|\right]^{2}+\left[n \pi+\frac{1}{2} \theta_{e}\right]^{2}+\mathcal{O}\left(\mathrm{e}^{-\left(k_{2}-\varepsilon\right) n}\right) \\
\left|\sqrt{\tau}-i \overline{\rho_{n}^{e}}\right|^{2} & =\left|\sqrt{\tau}+\frac{1}{2} \ln r-\left(n \pi+\frac{1}{2} \theta_{e}\right) i+\mathcal{O}\left(\mathrm{e}^{-k_{2} n}\right)\right|^{2} \\
& =\left[\sqrt{\tau}-\frac{1}{2}|\ln r|\right]^{2}+\left[n \pi+\frac{1}{2} \theta_{e}\right]^{2}+\mathcal{O}\left(\mathrm{e}^{-\left(k_{2}-\varepsilon\right) n}\right)
\end{aligned}
$$

there are $M_{2}, M_{3}>0$ such that

$$
\left|i \tau-\lambda_{n}^{e}\right|^{2}=\left|\sqrt{\tau}+\rho_{n}^{e}\right|^{2}\left|\sqrt{\tau}-\rho_{n}^{e}\right|^{2} \geq M_{2}\left[\tau+\left(n \pi+\frac{1}{2} \theta_{e}\right)^{2}\right]
$$

and

$$
\left|i \tau-\overline{\lambda_{n}^{e}}\right|^{2}=\left|\sqrt{\tau}+i \overline{\rho_{n}^{e}}\right|^{2}\left|\sqrt{\tau}-i \overline{\rho_{n}^{e}}\right|^{2} \geq M_{3}\left[\tau+\frac{1}{4}|\ln r|^{2}\right] .
$$

Hence, by (6.3) - (6.8), there is an $M>0$ such that

$$
\lim _{\tau \rightarrow \infty}|\tau|\|R(i \tau, \mathcal{A})\|^{2}=M<\infty .
$$

On the other hand, when $\tau \in \mathbb{R}$ and $\tau<0$, the same argument yields

$$
\begin{gathered}
|-i| \tau\left|-\lambda_{n}^{p}\right|^{2}=|\tau|^{2}+\left[n \pi+\frac{1}{2} \theta_{p}\right]^{4}+\mathcal{O}\left(\mathrm{e}^{-\left(k_{1}-\varepsilon\right) n}\right) \geq M_{1}|\tau|^{2}, \\
|-i| \tau\left|-\lambda_{n}^{e}\right|^{2}=|| \tau\left|-i \lambda_{n}^{e}\right|^{2}=|| \tau\left|-i^{2} \rho_{2 n}^{2}\right|^{2}=\left|\sqrt{|\tau|}+i \rho_{n}^{e}\right|^{2}\left|\sqrt{|\tau|}-i \rho_{n}^{e}\right|^{2} \\
\geq M_{3}\left[|\tau|+\frac{1}{4}|\ln r|^{2}\right]
\end{gathered}
$$


and

$$
\begin{aligned}
|-i| \tau\left|-\overline{\lambda_{n}^{e}}\right|^{2} & =|| \tau\left|-i \overline{\lambda_{n}^{e}}\right|^{2}=|| \tau\left|-\overline{\left(\rho_{n}^{e}\right)^{2}}\right|^{2}=\left|\sqrt{|\tau|}+\overline{\rho_{n}^{e}}\right|^{2}\left|\sqrt{|\tau|}-\overline{\rho_{n}^{e}}\right|^{2}, \\
& \geq M_{2}\left[|\tau|+\left(n \pi+\frac{1}{2} \theta_{e}\right)^{2}\right] .
\end{aligned}
$$

Hence, as $\tau \rightarrow-\infty$, we have

$$
\lim _{\tau \rightarrow-\infty}|\tau|\|R(i \tau, \mathcal{A})\|^{2}=M<\infty .
$$

Therefore, this together with (6.9) yields (6.1), and by Theorem 6.2, the semigroup e ${ }^{\mathcal{A} t}$, generated by $\mathcal{A}$, is of a Gevrey class $\delta>2$ with $t_{0}=0$.

Acknowledgements. The authors are grateful to the referees for their comments and suggestions to improving the paper.

\section{REFERENCES}

[1] B. Belinskiy and I. Lasiecka, Gevrey's and trace regularity of a semigroup associated with beam equation and non-monotone boundary conditions. J. Math. Anal. Appl. 332 (2007) 137-154.

[2] N.Dunford and J.T. Schwartz, Linear Operators, Part III. John Wiley \& Sons, Inc., New York-London-Sydney (1971).

[3] I.C. Gohberg and M.G. Krein, Introduction to the Theory of Linear Nonselfadjoint Operators. Vol. 18 of Trans. Math. Monogr. AMS Providence, Rhode Island (1969).

[4] B.Z. Guo, J.M. Wang and S.P. Yung, On the $C_{0}$-semigroup generation and exponential stability resulting from a shear force feedback on a rotating beam. Systems Control Lett. 54 (2005) 557-574.

[5] M. Krstic, Control of an unstable reaction-diffusion PDE with long input delay. Systems Control Lett. 58 (2009) $773-782$.

[6] M. Krstic, Delay Compensation for Nonlinear, Adaptive, and PDE Systems. Systems and Control: Foundations and Applications. Birkhäuser Boston, Inc., Boston, MA (2009).

[7] R.E. Langer, On the zeros of exponential sum and integrals. Bull. Amer. Math. Soc. 37 (1931) 213-239.

[8] I. Lasiecka, Mathematical control theory of coupled PDEs. Society for Industrial and Applied Mathematics. SIAM, Philadelphia, PA (2002).

[9] B.Ya. Levin, Lectures on Entire Functions. Vol. 150 of Trans. Math. Monogr. American Mathematical Society, Providence, Rhode Island (1996).

[10] J. Locker, Spectral Theory of Non-Self-Adjoint Two-Point Differential Operators. Vol.73 of Math. Surv. Monogr. American Mathematical Society, Providence, Rhode Island (2000).

[11] Z.H. Luo and B.Z. Guo, Shear force feedback control of a single link flexible robot with revolute joint. IEEE Trans. Automat. Control 42 (1997) 53-65.

[12] Z.H. Luo, B.Z. Guo and O. Morgül, Stability and Stabilization of Infinite dimensional Systems with Applications. SpringerVerlag, London (1999).

[13] M.A. Naimark, Linear Differential Operators. In vol. I. Frederick Ungar Publishing Company, New York (1967).

[14] M.R. Opmeer, Nuclearity of Hankel operators for ultradifferentiable control systems. Systems Control Lett. 57 (2008) 913-918.

[15] A. Pazy, Semigroups of Linear Operators and Applications to Partial Differential Equations. Springer-Verlag, New York (1983).

[16] A.A. Shkalikov, Boundary value problems for ordinary differential equations with a parameter in the boundary conditions. J. Soviet Math. 33 (1986) 1311-1342.

[17] M.A. Shubov, Generation of Gevrey class semigroup by non-selfadjoint Euler-Bernoulli beam model. Math. Methods Appl. Sci. 29 (2006) 2181-2199.

[18] S. Taylor, Gevrey Regularity of Solutions of Evolution Equations and Boundary Controllability, Gevrey Semigroups. Ph.D. thesis, School of Mathematics, University of Minnesota (1989).

[19] J.M. Wang and B.Z. Guo, Analyticity and dynamic behavior of a damped three-layer sandwich beam. J. Optim. Theory Appl. 137 (2008) 675-689.

[20] J.M. Wang, B.Z. Guo and B. Chentouf, Boundary feedback stabilization of a three-layer sandwich beam: Riesz basis approach. ESAIM: COCV 12 (2006) 12-34.

[21] J.M. Wang, B. Ren and M. Krstic, Stabilization and Gevrey regularity of a Schrödinger equation in boundary feedback with a heat equation. IEEE Trans. Automatic Control 57 (2012) 179-185.

[22] R.M. Young, An Introduction to Nonharmonic Fourier Series. Academic Press, Inc., London (2001).

[23] X. Zhang and E. Zuazua, Polynomial decay and control of a $1-d$ hyperbolic-parabolic coupled system. J. Differ. Equ. 204 (2004) 380-438.

[24] X. Zhang and E. Zuazua, Asymptotic behavior of a hyperbolic-parabolic coupled system arising in fluid-structure interaction. Internat. Ser. Numer. Math. 154 (2007) 445-455. 